\title{
QUANTIFIER ELIMINATION IN QUASIANALYTIC STRUCTURES VIA NON-STANDARD ANALYSIS
}

\author{
KRZYSZTOF JAN NOWAK
}

\begin{abstract}
The paper is a continuation of our earlier article where we developed a theory of active and non-active infinitesimals and intended to establish quantifier elimination in quasianalytic structures. That article, however, did not attain full generality, which refers to one of its results, namely the theorem on an active infinitesimal, playing an essential role in our non-standard analysis. The general case was covered in our subsequent preprint, which constitutes a basis for the approach presented here. We also provide a quasianalytic exposition of the results concerning rectilinearization of terms and of definable functions from our earlier research. It will be used to demonstrate a quasianalytic structure corresponding to a quasianalytic Denjoy-Carleman class which, unlike the classical analytic structure, does not admit quantifier elimination in the language of restricted quasianalytic functions augmented merely by the reciprocal function $1 / x$. More precisely, we construct a plane definable curve, which indicates both that the classical theorem by J. Denef and L. van den Dries as well as Łojasiewicz's theorem that every subanalytic curve is semianalytic are no longer true for quasianalytic structures. Besides rectilinearization of terms, our construction makes use of some theorems on power substitution for Denjoy-Carleman classes and on non-extendability of quasianalytic function germs. The last result relies on Grothendieck's factorization and open mapping theorems for (LF)-spaces.
\end{abstract}

\section{INTRODUCTION}

The paper is a continuation of our earlier article [20] devoted to quantifier elimination in quasianalytic structures, where we developed a theory of active and non-active infinitesimals in the non-standard models of the universal diagram of a given quasianalytic structure. This theory allowed us to simultaneously examine the exchange property and valuation property for terms in the language $\mathcal{L}$ of restricted quasianalytic functions augmented by rational powers and, eventually, to establish quantifier elimination and description of definable functions by $\mathcal{L}$-terms. That article, however, did not

2000 Mathematics Subject Classification. Primary: 03C10, 32S45, 26E10; Secondary: 03C64, 32B20, 14P15.

Key words and phrases. Quasianalytic structures, quantifier elimination, active infinitesimals, special cubes and modifications, valuation property, exchange property, rectilinearization of quasi-subanalytic functions, Denjoy-Carleman classes. 
attain full generality, which refers to one of its results, namely, the theorem on an active infinitesimal (op. cit., Theorem 4.4) playing an essential role in our non-standard analysis. The general case was covered in our subsequent preprint [22] which constitutes a basis for the approach presented here. Recently, quantifier elimination for generalized quasianalytic algebras was achieved by J.-P. Rolin and T. Servi [26] by means of other methods, an essential ingredient of which was a quasianalytic version of rectilinearization of definable sets.

We also provide a quasianalytic exposition of the results concerning rectilinearization of $\mathcal{L}$-terms and of definable functions from our article [21], which is a technique much more delicate in comparison to rectilinearization of definable sets. Finally, we shall use this technique to demonstrate a quasianalytic structure corresponding to a quasianalytic Denjoy-Carleman class which, unlike the classical analytic structure, does not admit quantifier elimination in the language of restricted quasianalytic functions augmented merely by the reciprocal function $1 / x$. The construction was given in our preprints [25]. This indicates that the classical theorem by J. Denef and L. van den Dries [6] does no longer hold for quasianalytic structures. More precisely, we construct a plane definable curve, which also shows that the classical theorem of Łojasiewicz [14] that every subanalytic curve is semianalytic is no longer true for quasianalytic structures. Besides rectilinearization of terms, our construction applies some theorems on power substitution for Denjoy-Carleman classes and on non-extendability of quasianalytic function germs.

The content of this paper falls into eight sections discussed briefly below. In Section 2, we provide an exposition of results on rectilinearization of $\mathcal{L}$ terms from our paper [21]. Section 3 presents several annotated results on infinitesimals (including the theorem on an active infinitesimal) from our paper [20] which are ingredients of one induction procedure, investigated in the next section. Section 5 establishes quantifier elimination and description of definable functions by $\mathcal{L}$-terms as well as general versions of the valuation property and rectilinearization of definable functions.

The purpose of the subsequent sections is to construct a counterexample to the aforementioned problem, formulated in [22], whether a quasianalytic structure admits quantifier elimination in the language augmented merely by the reciprocal function $1 / x$. The construction of a counterexample, given in Section 8, makes use of rectilinearization of terms as well as of 
two function-theoretic theorems about Denjoy-Carleman classes. The first, established in Section 6, is concerned with power substitution. The other, presented in Section 7, is a refinement of the non-extendability theorem given by $\mathrm{V}$. Thilliez [31], relying on Grothendieck's factorization and open mapping theorems for (LF)-spaces.

Let us recall (cf. $[19,20,23,24]$ ) that a quasianalytic structure $\mathbb{R}_{\mathcal{Q}}$ is the expansion of the real field by restricted $\mathcal{Q}$-analytic functions (abbreviated to $\mathcal{Q}$-functions) determined by a system $\mathcal{Q}=\left(\mathcal{Q}_{m}\right)_{m \in \mathbb{N}}$ of sheaves of local $\mathbb{R}$ algebras of smooth functions on $\mathbb{R}^{m}$, subject to the following six conditions:

(1) each algebra $\mathcal{Q}(U)$ contains the restrictions of polynomials;

(2) $\mathcal{Q}$ is closed under composition, i.e. the composition of $\mathcal{Q}$-maps is a $\mathcal{Q}$-map (whenever it is well defined);

(3) $\mathcal{Q}$ is closed under inverse, i.e. if $\varphi: U \longrightarrow V$ is a $\mathcal{Q}$-map between open subsets $U, V \subset \mathbb{R}^{n}, a \in U, b \in V$ and if $\partial \varphi / \partial x(a) \neq 0$, then there are neighbourhoods $U_{a}$ and $V_{b}$ of $a$ and $b$, respectively, and a $\mathcal{Q}$-diffeomorphism $\psi: V_{b} \longrightarrow U_{a}$ such that $\varphi \circ \psi$ is the identity map on $V_{b}$;

(4) $\mathcal{Q}$ is closed under differentiation;

(5) $\mathcal{Q}$ is closed under division by a coordinate, i.e. if a function $f \in \mathcal{Q}(U)$ vanishes for $x_{i}=a_{i}$, then $f(x)=\left(x_{i}-a_{i}\right) g(x)$ with some $g \in \mathcal{Q}(U)$;

(6) $\mathcal{Q}$ is quasianalytic, i.e. if $f \in \mathcal{Q}(U)$ and the Taylor series $\widehat{f}_{a}$ of $f$ at a point $a \in U$ vanishes, then $f$ vanishes in the vicinity of $a$.

Note that $\mathcal{Q}$-analytic maps (abbreviated to $\mathcal{Q}$-maps) give rise, in the ordinary manner, to the category $\mathcal{Q}$ of $\mathcal{Q}$-manifolds, which is a subcategory of that of smooth manifolds and smooth maps. Similarly, $\mathcal{Q}$-analytic, $\mathcal{Q}$-semianalytic and $\mathcal{Q}$-subanalytic sets can be defined. The above conditions ensure some (limited) resolution of singularities in the category $\mathcal{Q}$, including transformation to a normal crossing by blowing up (cf. [2, 3, 27]), upon which the geometry of quasianalytic structures relies; especially, in the absence of their good algebraic properties (cf. [27, 19, 20, 22]). Consequently, the structure $\mathbb{R}_{\mathcal{Q}}$ is model complete and o-minimal. Its definable subsets coincide with those subsets of $\mathbb{R}^{n}, n \in \mathbb{N}$, that are $\mathcal{Q}$-subanalytic in a semialgebraic compactification of $\mathbb{R}^{n}$. On the other hand, every polynomially bounded, o-minimal structure $\mathcal{R}$ determines a quasianalytic system of sheaves of germs of smooth functions that are locally definable in $\mathcal{R}$.

The examples of such categories are provided by quasianalytic DenjoyCarleman classes $\mathcal{Q}_{M}$, where $M=\left(M_{n}\right)_{n \in \mathbb{N}}$ are increasing sequences with $M_{0}=1$. The class $\mathcal{Q}_{M}$ consists of smooth functions $f(x)=f\left(x_{1}, \ldots, x_{m}\right)$ 
in $m$ variables, $m \in \mathbb{N}$, which are locally submitted to the following growth condition for their derivatives:

$$
\left|\partial^{|\alpha|} f / \partial x^{\alpha}(x)\right| \leq C \cdot R^{|\alpha|} \cdot|\alpha| ! \cdot M_{|\alpha|} \quad \text { for all } \quad \alpha \in \mathbb{N}^{n}
$$

with some constants $C, R>0$ depending only on the vicinity of a given point. This growth condition is often formulated in a slightly different way:

$$
\left|\partial^{|\alpha|} f / \partial x^{\alpha}(x)\right| \leq C \cdot R^{|\alpha|} \cdot M_{|\alpha|}^{\prime} \quad \text { for all } \alpha \in \mathbb{N}^{n},
$$

where $M_{n}^{\prime}=n ! M_{n}$. Obviously, the class $\mathcal{Q}_{M}$ contains the real analytic functions.

In order to ensure some important algebraic and analytic properties of the class $\mathcal{Q}_{M}$, it suffices to assume that the sequence $M$ or $M^{\prime}$ is log-convex. The latter implies that it is closed under multiplication (by virtue of the Leibniz formula). The former assumption is stronger and implies that it is closed under composition (Roumieu [28]) and under inverse (Komatsu [11]); see also [3]. Hence the set $\mathcal{Q}_{m}(M)$ of germs at $0 \in \mathbb{R}^{m}$ of $\mathcal{Q}_{M}$-analytic functions is a local ring. Then, moreover, the class $\mathcal{Q}_{M}$ is quasianalytic iff

$$
\sum_{n=0}^{\infty} \frac{M_{n}}{(n+1) M_{n+1}}=\infty
$$

(the Denjoy-Carleman theorem; see e.g. [29]), and is closed under differentiation and under division by a coordinate iff

$$
\sup _{n} \sqrt[n]{\left(\frac{M_{n+1}}{M_{n}}\right)}<\infty
$$

(cf. [16, 30]). It is well-known (cf. [4, 5, 30]) that, given two log-convex sequences $M$ and $N$, the inclusion $\mathcal{Q}_{M} \subset \mathcal{Q}_{N}$ holds iff there is a constant $C>0$ such that $M_{n} \leq C^{n} N_{n}$ for all $n \in \mathbb{N}$ or, equivalently,

$$
\sup \left\{\sqrt[n]{\frac{M_{n}}{N_{n}}}: n \in \mathbb{N}\right\}<\infty .
$$

\section{ReCTILINEARIZATION OF TERMS}

In our paper [21] we established several results concerning rectilinearization of functions definable by a Weierstrass system. It was done in two stages: first, we proved that every definable function is given piecewise by finitely many terms in the language augmented by rational powers; next, we proceed with rectilinearization of terms using transformation to a normal crossing by blowing up and induction with respect to the complexity of terms. That second stage can be repeated verbatim for the case of quasianalytic structures. We begin with suitable terminology. 
By a quadrant in $\mathbb{R}^{m}$ we mean a subset of $\mathbb{R}^{m}$ of the form:

$$
\begin{gathered}
\left\{x=\left(x_{1}, \ldots, x_{m}\right) \in \mathbb{R}^{m}: x_{i}=0, x_{j}>0, x_{k}<0\right. \\
\text { for } \left.i \in I_{0}, j \in I_{+}, k \in I_{-}\right\}
\end{gathered}
$$

where $\left\{I_{0}, I_{+}, I_{-}\right\}$is a partition of $\{1, \ldots, m\}$; its trace $Q$ on the cube $[-1,1]^{m}$ shall be called a bounded quadrant. The interior Int $(Q)$ of the quadrant $Q$ is its trace on the open cube $(-1,1)^{m}$. A bounded closed quadrant is the closure $\bar{Q}$ of a bounded quadrant $Q$, i.e. a subset of $\mathbb{R}^{m}$ of the form:

$$
\bar{Q}:=\left\{x \in[-1,1]^{m}: x_{i}=0, x_{j} \geq 0, x_{k} \leq 0 \quad \text { for } \quad i \in I_{0}, j \in I_{+}, k \in I_{-}\right\} .
$$

In this section, by a normal crossing on a bounded quadrant $Q$ in $\mathbb{R}^{m}$ we mean a function $g$ of the form

$$
g(x)=x^{\alpha} \cdot u(x)
$$

where $\alpha \in \mathbb{N}^{m}$ and $u$ is a function $\mathcal{Q}$-analytic near $\bar{Q}$ which vanishes nowhere on $\bar{Q}$. Below we state the theorem on rectilinearization of terms ([21, Theorem 1]) for the case of the quasianalytic structure $\mathbb{R}_{\mathcal{Q}}$.

Theorem 2.1. (Simultaneous Rectilinearization of $\mathcal{L}$-terms) If

$$
f_{1}, \ldots, f_{s}: \mathbb{R}^{m} \longrightarrow \mathbb{R}
$$

are functions given piecewise by a finite number of $\mathcal{L}$-terms, and $K$ is a compact subset of $\mathbb{R}^{m}$, then there exists a finite collection of modifications

$$
\varphi_{i}:[-1,1]^{m} \longrightarrow \mathbb{R}^{m}, \quad i=1, \ldots, p,
$$

such that

1) each $\varphi_{i}$ extends to a $\mathcal{Q}$-map in a neighbourhood of the cube $[-1,1]^{m}$, which is a composite of finitely many local blow-ups with smooth $\mathcal{Q}$-analytic centers and local power substitutions;

2) the union of the images $\varphi_{i}\left((-1,1)^{m}\right), i=1, \ldots, p$, is a neighbourhood of $K$;

3) for every bounded quadrant $Q_{j}, j=1, \ldots, 3^{m}$, the restriction to $Q_{j}$ of each function $f_{k} \circ \varphi_{i}, k=1, \ldots, s, i=1, \ldots, p$, either vanishes or is a normal crossing or a reciprocal normal crossing on $Q_{j}$.

Remark 2.2. Observe that, if the functions $f_{1}, \ldots, f_{s}$ are given piecewise by terms in the language of restricted $\mathcal{Q}$-analytic functions augmented merely by the reciprocal function $1 / x$, then one can require that the modifications $\varphi_{i}, i=1, \ldots, p$, be composite of finitely many local blow-ups with smooth $\mathcal{Q}$-analytic centers. 
We now recall the basic notions linked with our method of decomposition into special cubes, initiated in [19] and formulated in terms of special modifications in [20, Theorem 2.1]. Unless otherwise stated, we say that

$$
\varphi:(0,1)^{d} \longrightarrow S \subset \mathbb{R}^{m}
$$

is a special modification if the set $S$ is piecewise given by $\mathcal{L}$-terms, $\varphi$ is a $\mathcal{Q}$-map in the vicinity of $[0,1]^{d}$ which is a diffeomorphism of $(0,1)^{d}$ onto $S$, and the inverse map $\psi$ to this diffeomorphism is piecewise given by a finite number of $\mathcal{L}$-terms. Then $S$ is called a special cube with associated diffeomorphism $\varphi$.

Every bounded subset $F \subset \mathbb{R}^{m}$ given piecewise by $\mathcal{L}$-terms is a finite union of special cubes (op. cit., Corollary 2.3). Combined with Theorem 2.1, the method of special cubes allows us to obtain the following

Corollary 2.3. (Desingularization of $\mathcal{L}$-terms) If

$$
f_{1}, \ldots, f_{s}: \mathbb{R}^{m} \longrightarrow \mathbb{R}
$$

are bounded functions given piecewise by a finite number of $\mathcal{L}$-terms, and $K$ is a compact subset of $\mathbb{R}^{m}$, then there exists a finite collection of special modifications

$$
\sigma_{i}:(0,1)^{d_{i}} \longrightarrow S_{i} \subset \mathbb{R}^{m}, \quad i=1, \ldots, p,
$$

such that

1) the union of the special cubes $S_{i}, i=1, \ldots, p$, is a neighbourhood of $K$

2) each composite function $f_{k} \circ \varphi_{i}, k=1, \ldots, s$, extends to a $\mathcal{Q}$-function in the vicinity of $[0,1]^{d_{i}}$.

Proof. We proceed with induction with respect to the dimension $m$. The assertion is trivial if $m=0$. Supposing the assertion to hold for $d<m$, we shall prove it for $m$. Take the modifications

$$
\varphi_{i}:[-1,1]^{m} \longrightarrow \mathbb{R}^{m}, \quad i=1, \ldots, p,
$$

achieved in Theorem 2.1. For each $i=1, \ldots, p$, there exists a closed nowhere dense subset $V_{i} \subset \mathbb{R}^{m}$ given piecewise by $\mathcal{L}$-terms such that the restriction

$$
\varphi_{i}:(-1,1)^{m} \backslash \varphi_{i}^{-1}\left(V_{i}\right) \longrightarrow \mathbb{R}^{m} \backslash V_{i}
$$

is a diffeomorphism onto the image given piecewise by $\mathcal{L}$-terms, and the inverse map to this diffeomorphism is piecewise given by a finite number of $\mathcal{L}$-terms. By decomposition into special cubes [20, Theorem 2.1]), it is enough to consider the restrictions of the functions $f_{1}, \ldots, f_{s}$, to the subsets 
$V_{i}$ of dimensions $d_{i}<m, i=1, \ldots, p$. Again, application of special modifications reduces the problem to the dimensions $d_{i}<m$, which finishes the proof by the induction hypothesis.

Now, let $T$ be the universal diagram of the structure $\mathbb{R}_{\mathcal{Q}}$ in the language $\mathcal{L}$ of restricted quasianalytic functions augmented by rational powers, i.e. the set of all universal $\mathcal{L}$-sentences that are true in $\mathbb{R}_{\mathcal{Q}}$ ). Fix a model $\mathcal{R}$ of the universal theory $T$ in the language $\mathcal{L}$. Every $\mathcal{L}$-substructure of $\mathcal{R}$ is a model of $T$. We always regard the standard model $\mathbb{R}_{\mathcal{Q}}$ as a substructure of $\mathcal{R}$. Since the decompositions into special cubes are described by $\mathcal{L}$-terms (both a special modification $\varphi$ and its inverse $\psi$ ), they are preserved by passage to any model $\mathcal{R}$ of $T$ :

$$
F=\bigcup_{j} S_{j} \quad \Longrightarrow \quad F^{\mathcal{R}}=\bigcup_{j} S_{j}^{\mathcal{R}} .
$$

For simplicity of notation, we shall usually omit the superscript ${ }^{\mathcal{R}}$ referring to the interpretations in a model $\mathcal{R}$, which will not lead to confusion.

We say that infinitesimals $\lambda=\left(\lambda_{1}, \ldots, \lambda_{m}\right) \in \mathcal{R}$ are analytically dependent, if $\lambda$ lie in a special cube $S=\varphi\left((0,1)^{d}\right)$ with $d<m$. We call infinitesimals $\lambda$ analytically independent if they are not analytically dependent. Analytical independence is preserved, of course, under permutation of infinitesimals. We say that a subset $A$ in $\mathcal{R}$ is analytically independent if every finite subset $A$ in $\mathcal{R}$ consists of analytically independent infinitesimals. If $A \subset B$ and the set $B$ is analytically independent, so is $A$.

For a subset $A \subset \mathcal{R}$, let $\langle A\rangle$ denote the substructure of $\mathcal{R}$ generated by $A$. We shall see in the next two sections that span operation $\langle A\rangle$ satisfies the exchange property.

The convex hull of $\mathbb{R}$ in $\mathcal{R}$ is a valuation ring $V$ of bounded (with respect to $\mathbb{R}$ ) elements in $\mathcal{R}$; its maximal ideal $\mathfrak{m}$ consists of all infinitesimals in $\mathcal{R}$. The valuation $v$ induced by $V$ is called the standard valuation on the field $\mathcal{R}$; its value group $\Gamma_{\mathcal{R}}$ is a $\mathbb{Q}$-vector space. In order to investigate the valuation $v$, we have established in paper [20] several results about $\mathcal{Q}$ functions, recalled in this and the next section.

Now we are going to formulate rectilinearization of terms in the language of infinitesimals. Clearly, Corollary 2.3 immediately yields the version stated below, which coincides with that from op. cit., Corollary 2.6, whose proof, however, was provided too scantily. 
Corollary 2.4. Consider an $\mathcal{L}$-term $t(x)$ and positive analytically independent infinitesimals $\lambda=\left(\lambda_{1}, \ldots, \lambda_{m}\right)$. If $t(\lambda)$ is bounded, then there exist a special modification

$$
\varphi:(0,1)^{m} \longrightarrow \mathbb{R}^{m} \text { with } \lambda=\varphi\left(\lambda^{\prime}\right) \text { for some } \lambda^{\prime} \in(0,1)^{m}
$$

such that the superposition $f:=t \circ \varphi$ extends to a $\mathcal{Q}$-function in the vicinity of $[0,1]^{m}$; in particular we have $t(\lambda)=f\left(\lambda^{\prime}\right)$.

\section{Some RESUlts ON ACTIVE AND NON-ACTIVE INFINITESIMALS}

In paper [20], we developed a theory of active and non-active infinitesimals, crucial for our approach to the geometry of quasianalytic structures. Here, we wish to present several annotated results of this theory, which are ingredients of an induction procedure investigated in the next section. We say that an infinitesimal $\mu$ is non-active over infinitesimals $\lambda=\left(\lambda_{1}, \ldots, \lambda_{m}\right)$ if for each $\mathcal{L}$-term $t(x)$ we have

$$
v(\mu-t(\lambda)) \in \Gamma_{\langle\lambda\rangle} .
$$

Otherwise, the infinitesimal $\mu$ is called active over $\lambda$. It is clear that if $\mu$ is non-active over $\lambda$, so is the infinitesimal $\mu^{\prime}=s(\lambda) \mu+t(\lambda)$ that is the value at $(\lambda, \mu)$ of any $y$-linear $\mathcal{L}$-term.

We first examined, by means of successive lowering order of a given $\mathcal{Q}$ function (op. cit., Theorem 3.1), its behaviour at non-active infinitesimals (op. cit., Propositions 3.2 and 3.3), and thence conclude that, given a finite number of infinitesimals $\lambda=\left(\lambda_{1}, \ldots, \lambda_{m}\right)$, the value group $\Gamma_{\langle\lambda\rangle}$ is a vector space over $\mathbb{Q}$ of dimension $\leq m$ (op. cit., Corollary 3.4). Also examined were the behaviour of $\mathcal{L}$-terms (op. cit., Proposition 3.5) and the exchange property (op. cit., Proposition 3.6) at such infinitesimals. However, it was yet more difficult to describe the behaviour of $\mathcal{L}$-terms at active infinitesimals. To this end, we introduced the concept of a regular sequence of infinitesimals: a sequence $\lambda=\left(\lambda_{1}, \ldots, \lambda_{m}\right)$ of infinitesimals shall be called regular with main part $\lambda_{1}, \ldots, \lambda_{k}$, if the valuations

$$
v\left(\lambda_{1}\right), \ldots, v\left(\lambda_{k}\right) \in \Gamma_{\langle\lambda\rangle}
$$

form a basis over $\mathbb{Q}$ of the valuation group $\Gamma_{\left\langle\lambda_{1}, \ldots, \lambda_{m}\right\rangle}$.

Now we list five results from our paper [20], including the theorem on an active infinitesimal. Yet it has not been proven in full generality, nevertheless, covering the classical analytic case. Their formulations below take into account the number of infinitesimals under study, because these results will be encompassed by one induction procedure. 
$\left(\mathbf{I}_{\mathbf{m}}\right)$ (op. cit., Theorem 4.4 on an Active Infinitesimal)

For any $n \leq m$, consider a regular sequence $\mu, \lambda_{1}, \ldots, \lambda_{n}$ of infinitesimals with main part $\mu, \lambda_{1}, \ldots, \lambda_{k}$ and an $\mathcal{L}$-term $t(y, x), x=\left(x_{1} \ldots, x_{n}\right)$, such that

$$
\nu:=t(\mu, \lambda) \notin\langle\lambda\rangle
$$

is an infinitesimal. If $v(\mu) \notin \Gamma_{\langle\lambda\rangle}$, then $\nu$ is active over the infinitesimals $\lambda$.

$\left(\mathbf{I I}_{\mathbf{m}}\right)$ (op. cit., Proposition 4.7)

For any $n \leq m$, consider a regular sequence $\lambda_{1}, \ldots, \lambda_{n}$ of infinitesimals with main part $\lambda_{1}, \ldots, \lambda_{k}$ and an infinitesimal $\mu$ with $v(\mu) \notin \Gamma_{\langle\lambda\rangle}$. Then $\operatorname{dim} \Gamma_{\langle\mu, \lambda\rangle}=k+1$ whence $\mu, \lambda_{1}, \ldots, \lambda_{n}$ is a regular sequence of infinitesimals with main part $\mu, \lambda_{1}, \ldots, \lambda_{k}$.

$\left(\mathbf{I I I}_{\mathbf{m}}\right)$ (op. cit., Corollary 4.8; Valuation Property for $\mathcal{L}$-terms)

For any $n \leq m$ and infinitesimals $\mu, \lambda_{1}, \ldots, \lambda_{n}$, we have the following $d i$ chotomy:

- either $\mu$ is non-active over $\lambda$, and then $\Gamma_{\langle\lambda, \mu\rangle}=\Gamma_{\langle\lambda\rangle}$;

- or $\mu$ is active over $\lambda$, and then $\operatorname{dim} \Gamma_{\langle\lambda, \mu\rangle}=\operatorname{dim} \Gamma_{\langle\lambda\rangle}+1$.

In the latter case, one can find an $\mathcal{L}$-term $t(x)$ such that

$$
v(\mu-t(\lambda)) \notin \Gamma_{\langle\lambda\rangle} \quad \text { and } \quad \Gamma_{\langle\lambda, \mu\rangle}=\Gamma_{\langle\lambda\rangle} \oplus \mathbb{Q} \cdot v(\mu-t(\lambda)) .
$$

$\left(\mathbf{I V}_{\mathbf{m}}\right)$ (op. cit., Corollary 4.9; Exchange Property for $\mathcal{L}$-terms)

For any $n \leq m$ and infinitesimals $\mu, \lambda_{1}, \ldots, \lambda_{n}$, if $\nu \in\langle\lambda, \mu\rangle$ and $\nu \notin\langle\lambda\rangle$, then $\mu \in\langle\lambda, \nu\rangle$.

$\left(\mathbf{V}_{\mathbf{m}}\right)$ (op. cit., Proposition 5.1 and Corollary 5.2; Behaviour of Analytically Independent Infinitesimals)

For any $n \leq m$ and any two sets of analytically independent infinitesimals $\lambda_{1}, \ldots, \lambda_{n}$ and $\lambda_{1}^{\prime}, \ldots, \lambda_{n}^{\prime}$, if $\langle\lambda\rangle \subset\left\langle\lambda^{\prime}\right\rangle$, then $\langle\lambda\rangle=\left\langle\lambda^{\prime}\right\rangle$. Consequently, the infinitesimals $\left(\lambda_{1}, \ldots, \lambda_{n}, \mu\right)$ are analytically independent iff $\mu \notin\langle\lambda\rangle$.

The proofs of the above results provided in paper [20] indicate the following inferences:

$$
\left(\mathbf{I}_{\mathbf{m}}\right) \Longrightarrow\left(\mathbf{I I}_{\mathbf{m}+\mathbf{1}}\right), \quad\left(\mathbf{I I}_{\mathbf{m}}\right) \Longrightarrow\left(\mathbf{I I I}_{\mathbf{m}}\right)
$$

and

$$
\left(\mathbf{I}_{\mathbf{m}}\right) \wedge\left(\mathbf{I I I}_{\mathbf{m}}\right) \Longrightarrow\left(\mathbf{I V}_{\mathbf{m}}\right) \Longrightarrow\left(\mathbf{V}_{\mathbf{m}+\mathbf{1}}\right)
$$


Therefore the induction hypothesis $\left(\mathbf{I}_{\mathbf{m}-\mathbf{1}}\right)$ implies the hypotheses $\left(\mathbf{I I}_{\mathbf{m}}\right)$, $\left(\mathbf{I I I}_{\mathbf{m}}\right),\left(\mathbf{I V}_{\mathbf{m}-\mathbf{1}}\right)$ and $\left(\mathbf{V}_{\mathbf{m}}\right)$. Actually, in the proof we shall make use of the assertions $\left(\mathbf{I}_{\mathbf{m}-\mathbf{1}}\right),\left(\mathbf{I I I}_{\mathbf{m}}\right)$ and $\left(\mathbf{V}_{\mathbf{m}}\right)$.

Remark 3.1. The exchange property for $\mathcal{L}$-terms means exactly that the structure $\mathcal{R}$ with span operation is geometric, and that we have at our disposal the concepts of rank and basis for its substructures.

\section{Proof of the theorem on an ACtive infinitesimal}

We proceed with induction with respect to the number $m$ of infinitesimals $\lambda_{1}, \ldots, \lambda_{m}$. When $m=0$, then $\langle\lambda\rangle=\langle\emptyset\rangle=\mathbb{R}$ and the conclusion is evident. So take $m>0$ and assume the theorem holds when the number of infinitesimals $\lambda$ is smaller than $m$.

In [20], we have reduced the problem to the case where

$$
t(u, v, \tilde{x})=f(u, v / u, \tilde{x}), \quad \nu:=t(\mu, \lambda)=f\left(\mu, \lambda_{1} / \mu, \tilde{\lambda}\right),
$$

where $f(u, v, \tilde{x})$ is a function $\mathcal{Q}$-analytic at $0 \in \mathbb{R}^{m+1}$; here $v=x_{1}, \tilde{x}=$ $\left(x_{2}, \ldots, x_{m}\right)$ and $\tilde{\lambda}=\left(\lambda_{2} \ldots, \lambda_{m}\right)$.

The valuation group $\Gamma_{\langle\lambda, \mu\rangle}$ is a vector space over $\mathbb{Q}$ of dimension $\leq(m+1)$ (op.cit., Corollary 3.4). It is a direct sum of finitely many archimedean subgroups

$$
\Gamma_{\langle\lambda, \mu\rangle}=G_{1} \oplus \ldots \oplus G_{r} \quad \text { with } \quad G_{1}^{+}>\ldots>G_{r}^{+},
$$

where $G_{i}^{+}$stands for the semigroup of all positive elements of $G_{i}$. It is wellknown that every archimedean ordered abelian group is isomorphic to a subgroup of the ordered additive group $\mathbb{R}$ of real numbers.

Let $\varepsilon=\left(\varepsilon_{1}, \ldots, \varepsilon_{p}\right)$ denote those infinitesimals from among $\lambda$ for which

$$
v\left(\varepsilon_{1}\right), \ldots, v\left(\varepsilon_{p}\right)>G_{2} \oplus \ldots \oplus G_{r}
$$

and $\delta=\left(\delta_{1}, \ldots, \delta_{q}\right)$ the remaining $\lambda$ 's; obviously, $p+q=m$. The valued field $\langle\lambda, \mu\rangle$ can be completed with respect to the standard valuation $v$, and the completion has the same valuation group. Notice that the topology induced by $v$ is metrizable with the basis of zero neighbourhoods consisting of sets of the form

$$
\{t(\mu, \delta, \varepsilon): v(t(\mu, \delta, \varepsilon))>\gamma\}, \quad \gamma \in G_{1} .
$$

In the completion, one can deal with formal power series in the infinitesimals $\varepsilon$ with $\mathcal{Q}$-analytic coefficients taken on the infinitesimals $\delta$.

We encounter two cases:

Case $A$, where $\lambda_{1}$ is one of the $\varepsilon$ 's, say $\lambda_{1}=\varepsilon_{1}$; 
or

Case $B$, where $\lambda_{1}$ is one of the $\delta$ 's, say $\lambda_{1}=\delta_{1}$.

CASE A. Consider the Taylor coefficients

$$
\frac{1}{i ! j !} \cdot \frac{\partial^{i+j} f}{\partial u^{i} \partial v^{j}}(0,0, \tilde{x})=: a_{i j}(\tilde{x}), \quad i, j \in \mathbb{N}
$$

which are $\mathcal{Q}$-analytic functions at zero. A crucial role is played by the following

Lemma 4.1. Under the assumptions of the theorem on an active infinitesimal $\left(\mathbf{I}_{\mathbf{m}}\right)$, we must have

$$
\sum_{i=0}^{\infty} a_{i, i+s}(\tilde{\lambda}) \cdot \varepsilon_{1}^{i} \neq 0 \quad \text { or } \quad \sum_{j=0}^{\infty} a_{j+s, j}(\tilde{\lambda}) \cdot \varepsilon_{1}^{j} \neq 0
$$

for some $s \in \mathbb{N} \backslash\{0\}$.

Proof. Suppose Lemma 4.1 were false. Then

$$
\sum_{i=0}^{\infty} a_{i, i+s}(\tilde{\lambda}) \cdot \varepsilon_{1}^{i}=0 \quad \text { and } \quad \sum_{j=0}^{\infty} a_{j+s, j}(\tilde{\lambda}) \cdot \varepsilon_{1}^{j}=0
$$

for all $s \in \mathbb{N} \backslash\{0\}$.

But we can find a model of the universal diagram $T$ with the infinitesimals $\lambda$ and an infinitesimal $\mu^{*}$ such that $\lambda, \mu^{*}$ are analytically independent and

$$
v\left(\mu^{*}\right), v\left(\varepsilon_{1} / \mu^{*}\right)>G_{2} \oplus \ldots \oplus G_{r} .
$$

Indeed, it follows from the induction hypothesis that the assertion $\left(\mathbf{V}_{\mathbf{m}}\right)$ holds. The infinitesimals $\lambda$ and $\mu^{*}$ are therefore analytically independent iff $\mu^{*} \notin\langle\lambda\rangle$. Consequently, it suffices to find a model of the universal diagram $T$ along with the diagram of the structure $\langle\lambda\rangle$ and the sentences of the form

$$
c \neq t(\lambda) \quad \text { where } t(x) \text { are } \mathcal{L} \text {-terms }
$$

and the sentence

$$
\frac{1}{2} \sqrt{\varepsilon_{1}}<c<2 \sqrt{\varepsilon_{1}} ;
$$

here $c$ denotes a new constant construed as $\mu^{*}$. Its existence can be immediately deduced through model-theoretic compactness.

Under the conditions stated above, we have

$$
t\left(\mu^{*}, \lambda\right)=f\left(\mu^{*}, \lambda_{1} / \mu^{*}, \tilde{\lambda}\right)=\sum_{i=0}^{\infty} a_{i, i}(\tilde{\lambda}) \cdot \varepsilon_{1}^{i}+
$$




$$
\begin{gathered}
+\sum_{s=1}^{\infty}\left(\varepsilon_{1} / \mu^{*}\right)^{s} \cdot \sum_{i=0}^{\infty} a_{i, i+s}(\tilde{\lambda}) \cdot \varepsilon_{1}^{i}+\sum_{s=1}^{\infty}\left(\mu^{*}\right)^{s} \cdot \sum_{j=0}^{\infty} a_{j+s, j}(\tilde{\lambda}) \cdot \varepsilon_{1}^{i}= \\
=\sum_{i=0}^{\infty} a_{i, i}(\tilde{\lambda}) \cdot \varepsilon_{1}^{i},
\end{gathered}
$$

and thus we get

$$
\partial t / \partial u\left(\mu^{*}, \lambda\right)=0
$$

Here

$$
\partial t / \partial u(u, x)=\partial / \partial u f\left(u, x_{1} / u, \tilde{x}\right)
$$

is a $\mathcal{Q}$-function on an open set

$$
\Omega=S \times\left\{\tilde{x} \in \mathbb{R}^{m-1}:\left|x_{2}\right|, \ldots,\left|x_{m}\right|<\rho\right\},
$$

where $\rho \in \mathbb{R}, \rho>0$ is small enough, and

$$
S=\left\{\left(u, x_{1}\right) \in \mathbb{R}^{2}:|u|<\rho,\left|x_{1}\right|<\rho|u|\right\}
$$

is a sector. Since the infinitesimals $\mu^{*}, \lambda$ are analytically independent, the above $\mathcal{Q}$-function must vanish on an open special cube contained in $\Omega$. By the identity principle for quasianalytic functions, it vanishes identically on $\Omega$, and thus

$$
t(u, x)=f\left(u, x_{1} / u, \tilde{x}\right)=g(x),
$$

where $r \in \mathbb{R}, r \in(0, \rho)$, and $g(x):=f\left(r, x_{1} / r, \tilde{x}\right)$ is a $\mathcal{Q}$-function at $0 \in \mathbb{R}_{x}^{m}$. Hence

$$
\nu:=t(\mu, \lambda)=g(\lambda) \in\langle\lambda\rangle,
$$

and this contradiction completes the proof of Lemma 4.1.

Since $\mu \cdot \varepsilon_{1} / \mu=\varepsilon_{1}$, we see that

$$
v(\mu) \geq 1 / 2 v\left(\varepsilon_{1}\right) \quad \text { or } \quad v\left(\varepsilon_{1} / \mu\right) \geq 1 / 2 v\left(\varepsilon_{1}\right) .
$$

By symmetry, we may assume that the former condition holds. Then the series

$$
\begin{gathered}
\nu_{0}:=\sum_{i=j=0}^{\infty} a_{i j}(\tilde{\lambda}) \cdot \mu^{i} \cdot\left(\frac{\varepsilon_{1}}{\mu}\right)^{j}=\sum_{i=0}^{\infty} a_{i i}(\tilde{\lambda}) \cdot \varepsilon_{1}^{i}, \\
\nu_{+}:=\sum_{i=0}^{\infty} \sum_{j<i} a_{i j}(\tilde{\lambda}) \cdot \mu^{i} \cdot\left(\frac{\varepsilon_{1}}{\mu}\right)^{j}=\sum_{i=0}^{\infty} \sum_{j<i} a_{i j}(\tilde{\lambda}) \cdot \mu^{i-j} \cdot \varepsilon_{1}^{j}
\end{gathered}
$$

and

$$
\begin{aligned}
\nu_{-}:= & \sum_{i=0}^{\infty}\left(\frac{1}{i !} \cdot \frac{\partial^{i} f}{\partial u^{i}}\left(0, \frac{\varepsilon_{1}}{\mu}, \tilde{\lambda}\right) \cdot \mu^{i}-\sum_{j \leq i} a_{i j}(\tilde{\lambda}) \cdot \mu^{i} \cdot\left(\frac{\varepsilon_{1}}{\mu}\right)^{j}\right)= \\
& =\sum_{i=0}^{\infty}\left(\frac{1}{i !} \cdot \frac{\partial^{i} f}{\partial u^{i}}\left(0, \frac{\varepsilon_{1}}{\mu}, \tilde{\lambda}\right)-\sum_{j \leq i} a_{i j}(\tilde{\lambda}) \cdot\left(\frac{\varepsilon_{1}}{\mu}\right)^{j}\right) \cdot \mu^{i}
\end{aligned}
$$


are well defined elements of the completion of the valued field $\langle\lambda, \mu\rangle$ with respect to the standard valuation $v$. We have $\nu=\nu_{+}+\nu_{0}+\nu_{-}$. Observe that the $\mathcal{Q}$-analytic function which occurs in the $i$-th summand of the last series is of the form

$$
\left(\frac{1}{i !} \cdot \frac{\partial^{i} f}{\partial u^{i}}(0, v, \tilde{x})-\sum_{j \leq i} a_{i j}(\tilde{x}) \cdot v^{j}\right) \cdot u^{i}=g_{i}(v, \tilde{x}) \cdot v^{i+1} \cdot u^{i}
$$

for some function $g_{i} \mathcal{Q}$-analytic at zero.

Lemma 4.2. If

$$
\sum_{i=0}^{\infty} a_{i, i+s}(\tilde{\lambda}) \cdot \varepsilon_{1}^{i} \neq 0 \quad \text { or } \quad \sum_{j=0}^{\infty} a_{j+s, j}(\tilde{\lambda}) \cdot \varepsilon_{1}^{j} \neq 0
$$

for some $s \in \mathbb{N} \backslash\{0\}$, then we have, respectively

$$
\nu_{-} \neq 0 \quad \text { and } \quad v\left(\nu_{-}\right) \in \Gamma_{\langle\lambda\rangle}-(\mathbb{N} \backslash\{0\}) \cdot v(\mu)
$$

or

$$
\nu_{+} \neq 0 \text { and } v\left(\nu_{+}\right) \in \Gamma_{\langle\lambda\rangle}+(\mathbb{N} \backslash\{0\}) \cdot v(\mu) .
$$

Proof. Consider first the latter case. Clearly,

$$
\nu_{+}=\sum_{l=1}^{\infty}\left(\sum_{j=0}^{\infty} a_{j+l, j}(\tilde{\lambda}) \cdot \varepsilon_{1}^{j}\right) \cdot \mu^{l},
$$

and the values of (the valuation $v$ taken on) the $l$-th summands of the above series are pairwise distinct, unless they are infinity. Consequently, $v\left(\nu_{+}\right)$is the minimum of the values of those summands, because some summands are non-vanishing (for instance, the $s$-th one). Hence

$$
v\left(\nu_{+}\right)<\infty \text { and } v\left(\nu_{+}\right) \in \Gamma_{\langle\lambda\rangle}+(\mathbb{N} \backslash\{0\}) \cdot v(\mu),
$$

as desired.

In the former case, take $n$ large enough so that

$$
v\left(\mu^{n}\right)>v\left(\sum_{i=0}^{\infty} a_{i, i+s}(\tilde{\lambda}) \cdot \mu^{i} \cdot\left(\frac{\varepsilon_{1}}{\mu}\right)^{i+s}\right),
$$

and write down $\nu_{-}$as follows:

$$
\begin{gathered}
\nu_{-}=\sum_{l=1}^{s}\left(\sum_{i=0}^{\infty} a_{i, i+l}(\tilde{\lambda}) \cdot \mu^{i} \cdot\left(\frac{\varepsilon_{1}}{\mu}\right)^{i+l}\right)+ \\
+\sum_{i=0}^{n-1}\left(\frac{1}{i !} \cdot \frac{\partial^{i} f}{\partial u^{i}}\left(0, \frac{\varepsilon_{1}}{\mu}, \tilde{\lambda}\right)-\sum_{j \leq i+s} a_{i j}(\tilde{\lambda}) \cdot\left(\frac{\varepsilon_{1}}{\mu}\right)^{j}\right) \cdot \mu^{i}+ \\
+\sum_{i=n}^{\infty}\left(\frac{1}{i !} \cdot \frac{\partial^{i} f}{\partial u^{i}}\left(0, \frac{\varepsilon_{1}}{\mu}, \tilde{\lambda}\right)-\sum_{j \leq i+s} a_{i j}(\tilde{\lambda}) \cdot\left(\frac{\varepsilon_{1}}{\mu}\right)^{j}\right) \cdot \mu^{i}
\end{gathered}
$$


Observe again that the $\mathcal{Q}$-analytic functions which occur in the $i$-th summands of the second series above are of the form

$$
\left(\frac{1}{i !} \cdot \frac{\partial^{i} f}{\partial u^{i}}(0, v, \tilde{x})-\sum_{j \leq i+s} a_{i j}(\tilde{x}) \cdot v^{j}\right) \cdot u^{i}=h_{i}(v, \tilde{x}) \cdot v^{i+s+1} \cdot u^{i}
$$

for some functions $h_{i} \mathcal{Q}$-analytic at zero, $i=0, \ldots, n$. Hence

$$
\begin{gathered}
\sum_{i=0}^{n-1}\left(\frac{1}{i !} \cdot \frac{\partial^{i} f}{\partial u^{i}}\left(0, \frac{\varepsilon_{1}}{\mu}, \tilde{\lambda}\right)-\sum_{j \leq i+s} a_{i j}(\tilde{\lambda}) \cdot\left(\frac{\varepsilon_{1}}{\mu}\right)^{j}\right) \cdot \mu^{i}= \\
=\left(\frac{\varepsilon_{1}}{\mu}\right)^{s+1} \cdot \sum_{i=0}^{n} h_{i}\left(\frac{\varepsilon_{1}}{\mu}, \tilde{\lambda}\right) \cdot \varepsilon_{1}^{i} .
\end{gathered}
$$

By op. cit., Corollary 2.11, we have

$$
v\left(h_{i}\left(\frac{\varepsilon_{1}}{\mu}, \tilde{\lambda}\right)\right) \in \Gamma_{\langle\lambda\rangle} \oplus \mathbb{N} \cdot v\left(\frac{\varepsilon_{1}}{\mu}\right) .
$$

Since

$$
\sum_{i=0}^{\infty} a_{i, i+l}(\tilde{\lambda}) \cdot \mu^{i} \cdot\left(\frac{\varepsilon_{1}}{\mu}\right)^{i+l}=\left(\frac{\varepsilon_{1}}{\mu}\right)^{l} \cdot \sum_{i=0}^{\infty} a_{i, i+l}(\tilde{\lambda}) \cdot \varepsilon_{1}^{i}, \quad l=1, \ldots, s,
$$

the values

$$
v\left(\sum_{i=0}^{\infty} a_{i, i+l}(\tilde{\lambda}) \cdot \mu^{i} \cdot\left(\frac{\varepsilon_{1}}{\mu}\right)^{i+l}\right), \quad l=1, \ldots, s,
$$

and

$$
v\left(\sum_{i=0}^{n-1}\left(\frac{1}{i !} \cdot \frac{\partial^{i} f}{\partial u^{i}}\left(0, \frac{\varepsilon_{1}}{\mu}, \tilde{\lambda}\right)-\sum_{j \leq i+s} a_{i j}(\tilde{\lambda}) \cdot\left(\frac{\varepsilon_{1}}{\mu}\right)^{j}\right) \cdot \mu^{i}\right)
$$

are pairwise distinct, unless they are infinity. Consequently, $v\left(\nu_{-}\right)$is the minimum of the above $(s+1)$ values. Hence

$$
v\left(\nu_{-}\right)<\infty \text { and } v\left(\nu_{-}\right) \in \Gamma_{\langle\lambda\rangle}-(\mathbb{N} \backslash\{0\}) \cdot v(\mu),
$$

which completes the proof of Lemma 4.2.

Now, take $n \in \mathbb{N}$ large enough so that

$$
n \cdot v\left(\varepsilon_{1}\right)>\min \left\{v\left(\nu_{-}\right), v\left(\nu_{+}\right)\right\} .
$$

Then

$$
v\left(\nu-\sum_{i=0}^{n-1} a_{i, i}(\tilde{\lambda}) \cdot \varepsilon_{1}^{i}\right)=\min \left\{v\left(\nu_{-}\right), v\left(\nu_{+}\right)\right\} \notin \Gamma_{\langle\lambda\rangle} .
$$

This means that $\nu$ is active over the infinitesimals $\lambda$, concluding the proof of the theorem on an active infinitesimal $\left(\mathbf{I}_{\mathbf{m}}\right)$ in Case A. 
CASE B. Let us rename the coordinates in $\mathbb{R}^{m}$ in the following way: the coordinates $x=\left(x_{1}, \ldots, x_{q}\right)$ correspond to the infinitesimals $\delta$ and the coordinates $y=\left(y_{1}, \ldots, y_{p}\right)$ correspond to the infinitesimals $\varepsilon$. In the new variables, the function $f$ can be written down as $f(u, v, \tilde{x}, y), \tilde{x}=\left(x_{2}, \ldots, x_{q}\right)$. We first establish the following

Reduction Step. We can assume that the infinitesimal $\nu$ is of the form $\nu=f\left(\mu, \delta_{1} / \mu, \tilde{\delta}, \varepsilon\right)$, where $f$ is a function $\mathcal{Q}$-analytic at zero, and

$$
v(\varepsilon)>G_{2} \oplus \ldots \oplus G_{r} \quad \text { and } \quad \Gamma_{\langle\delta, \mu\rangle}<G_{1}^{+} .
$$

Proof. First, we recursively attach the old infinitesimals $\delta_{2}, \ldots, \delta_{q}$, after performing suitable special modifications, either to the new infinitesimals $\delta^{\prime}$ or to the new infinitesimals $\varepsilon^{\prime}$, so as to fulfil the conditions

$$
v\left(\varepsilon^{\prime}\right)>G_{2} \oplus \ldots \oplus G_{r} \quad \text { and } \quad \Gamma_{\left\langle\delta^{\prime}\right\rangle}<G_{1}^{+} .
$$

At the beginning, take as new infinitesimals $\delta^{\prime}$ those infinitesimals from $\delta_{2}, \ldots, \delta_{q}$, which lie in the main part of the regular sequence $\mu, \lambda_{1}, \ldots, \lambda_{k}$. Having constructed a sequence $\delta_{2}^{\prime}, \ldots, \delta_{i}^{\prime}$, take an infinitesimal $\delta_{j}$ from among $\delta_{2}, \ldots, \delta_{q}$, which has not yet been considered in the process. If

$$
\Gamma_{\left\langle\delta_{2}^{\prime}, \ldots, \delta_{i}^{\prime}, \delta_{j}\right\rangle}<G_{1}^{+},
$$

attach $\delta_{j}=: \delta_{i+1}^{\prime}$ to the new infinitesimals $\delta^{\prime}$. Otherwise $\delta_{j}$ is active over $\delta_{2}^{\prime}, \ldots, \delta_{i}^{\prime}$. By the valuation property $\left(\mathbf{I I I}_{\mathbf{m}}\right)$, which holds by the induction hypothesis, there is an $\mathcal{L}$-term $\tau\left(\delta_{2}^{\prime}, \ldots, \delta_{i}^{\prime}\right)$ such that

$$
v\left(\delta_{j}-\tau\left(\delta_{2}^{\prime}, \ldots, \delta_{i}^{\prime}\right)\right)>G_{2} \oplus \ldots \oplus G_{r} .
$$

Via desingularization of $\mathcal{L}$-terms (Corollary 2.4 and [20, Corollary 2.6]), we can assume, after a suitable change of the infinitesimals $\delta_{2}^{\prime}, \ldots, \delta_{i}^{\prime}$ by special modification, that

$$
\tau\left(\delta_{2}^{\prime}, \ldots, \delta_{i}^{\prime}\right)=\varphi\left(\delta_{2}^{\prime}, \ldots, \delta_{i}^{\prime}\right),
$$

where $\varphi$ is a function $\mathcal{Q}$-analytic at zero. Then we attach the infinitesimal

$$
\omega:=\delta_{j}-\varphi\left(\delta_{2}^{\prime}, \ldots, \delta_{i}^{\prime}\right)
$$

to the new infinitesimals $\varepsilon$. By substitution $\omega+\varphi\left(\delta_{2}^{\prime}, \ldots, \delta_{i}^{\prime}\right)$ for $\delta_{j}$, we are done. We continue this process until all infinitesimals $\delta_{2}, \ldots, \delta_{q}$ have been considered. In this manner, we get infinitesimals $\delta_{2}^{\prime}, \ldots, \delta_{t}^{\prime}$ and $\epsilon_{1}^{\prime}, \ldots, \epsilon_{i}^{\prime}$ that fulfil conditions 4.1 .

Next, consider the infinitesimal $\delta_{1}$. If

$$
\Gamma_{\left\langle\delta_{2}^{\prime}, \ldots, \delta_{t}^{\prime}, \delta_{1}\right\rangle}<G_{1}^{+},
$$


we are done by putting $\delta_{1}^{\prime}:=\delta_{1}$. Otherwise $\delta_{1}$ is active over $\delta_{2}^{\prime}, \ldots, \delta_{t}^{\prime}$. As before, by the valuation property $\left(\mathbf{I I I}_{\mathbf{m}}\right)$ and via desingularization of $\mathcal{L}$-terms, we can assume that

$$
v(\omega)>G_{2} \oplus \ldots \oplus G_{r} \quad \text { with } \quad \omega:=\delta_{1}-\varphi\left(\delta_{2}^{\prime}, \ldots, \delta_{t}^{\prime}\right),
$$

where $\varphi$ is a function $\mathcal{Q}$-analytic at zero. Let $\delta^{\prime}=\left(\delta_{2}^{\prime}, \ldots, \delta_{t}^{\prime}\right)$. Then, similarly to op.cit., Section 4, we can replace the function $f$ by some other $\mathcal{Q}$-analytic functions as follows:

$$
\begin{gathered}
\nu=f\left(\mu,\left(\varphi\left(\delta^{\prime}\right)+\omega\right) / \mu, \delta^{\prime}, \varepsilon^{\prime}\right)=f_{1}\left(\mu, \varphi\left(\delta^{\prime}\right) / \mu, \omega / \mu, \delta^{\prime}, \varepsilon^{\prime}\right)= \\
=f_{2}\left(\mu, \varphi\left(\delta^{\prime}\right) / \mu, \omega / \varphi\left(\delta^{\prime}\right), \delta^{\prime}, \varepsilon^{\prime}\right) .
\end{gathered}
$$

We can thus attach the infinitesimal $\varepsilon_{s+1}^{\prime}:=\omega / \varphi\left(\delta^{\prime}\right)$ to the infinitesimals $\varepsilon^{\prime}$, and then

$$
\nu=f_{2}\left(\mu, \varphi\left(\delta^{\prime}\right) / \mu, \varepsilon_{s+1}^{\prime}, \delta^{\prime}, \varepsilon^{\prime}\right) .
$$

For simplicity, we drop the sign of apostrophe over the name of infinitesimals. By transforming the function $\varphi$ to a normal crossing, we may assume that

$$
\nu=f_{3}\left(\mu, \delta^{\alpha} / \mu, \delta, \varepsilon\right)
$$

for some $\alpha \in \mathbb{N}^{q}$. Replacing the infinitesimals $\mu$ and $\delta$ by their suitable roots, we may assume that $\delta^{\alpha}=\delta_{1} \cdot \ldots \cdot \delta_{k}$ for some $k \leq q$. Now, we can successively lower the number $k$ of these factors as follows. Since $v(\mu) \notin \Gamma_{\langle\delta\rangle}$, exactly one of the two fractions $\delta_{1} \cdot \ldots \cdot \delta_{k-1} / \mu$ or $\mu / \delta_{1} \cdot \ldots \cdot \delta_{k-1}$ is an infinitesimal. In the former case, we get

$$
\nu=f_{3}\left(\mu, \delta_{1} \cdot \ldots \cdot \delta_{k} / \mu, \delta, \varepsilon\right)=f_{4}\left(\mu, \delta_{1} \cdot \ldots \cdot \delta_{k-1} / \mu, \delta, \varepsilon\right) ;
$$

and in the latter

$$
\begin{gathered}
\nu=f_{3}\left(\mu, \delta_{1} \cdot \ldots \cdot \delta_{k} / \mu, \delta, \varepsilon\right)=f_{4}\left(\mu, \mu / \delta_{1} \cdot \ldots \cdot \delta_{k-1}, \delta_{1} \cdot \ldots \cdot \delta_{k} / \mu, \delta, \varepsilon\right)= \\
=f_{5}\left(\mu / \delta_{1} \cdot \ldots \cdot \delta_{k-1}, \delta_{1} \cdot \ldots \cdot \delta_{k} / \mu, \delta, \varepsilon\right),
\end{gathered}
$$

and thus, replacing $\mu$ by $\mu^{\prime}:=\mu / \delta_{1} \cdot \ldots \cdot \delta_{k-1}$, we get

$$
\nu=f_{5}\left(\mu^{\prime}, \delta_{k} / \mu^{\prime}, \delta, \varepsilon\right) \text {. }
$$

Again, we drop the sign of apostrophe. Eventually, we can assume that

$$
\nu=f_{6}\left(\mu, \delta_{1} / \mu, \delta_{1}, \tilde{\delta}, \varepsilon\right)=f_{7}\left(\mu, \delta_{1} / \mu, \tilde{\delta}, \varepsilon\right),
$$

which is the desired result.

Summing up, the above construction yields a new regular sequence of infinitesimals $\delta, \varepsilon$ which satisfy conditions 4.1. To finish the reduction step, we must still show that $\Gamma_{\langle\delta, \mu\rangle}<G_{1}^{+}$. But this follows immediately from 
the valuation property $\left(\mathbf{I I I}_{\mathbf{m}}\right)$, which is at our disposal by the induction hypothesis, applied to the infinitesimals $\delta$ and $\mu$.

For the rest of the proof of $\left(\mathbf{I}_{\mathbf{m}}\right)$, we shall keep the conditions established in the reduction step. In the completion of the valued field $\langle\lambda, \mu\rangle$ with respect to the standard valuation $v$, we can present the infinitesimal $\nu$ in the following form:

$$
\nu=f\left(\mu, \delta_{1} / \mu, \tilde{\delta}, \varepsilon\right)=\sum_{\alpha \in \mathbb{N}^{p}} \varepsilon^{\alpha} \cdot f_{\alpha}\left(\mu, \delta_{1} / \mu, \tilde{\delta}\right),
$$

where

$$
f_{\alpha}(u, v, \tilde{x}):=\frac{1}{\alpha !} \cdot \frac{\partial^{|\alpha|} f}{\partial y^{\alpha}}(u, v, \tilde{x}, 0), \quad \alpha \in \mathbb{N}^{p} .
$$

We need an elementary fact about the standard valuation $v$.

Lemma 4.3. Consider a finite number of elements $g_{i}, h_{i} \in\langle\lambda, \mu\rangle, i=$ $1, \ldots, n$, such that

$$
v\left(h_{1}\right), \ldots, v\left(h_{n}\right)>G_{2} \oplus \ldots \oplus G_{r}
$$

and

$$
v\left(\sum_{i=1}^{n} c_{i} \cdot g_{i}\right)<G_{1}^{+}, \quad c_{i} \in \mathbb{R}, i=1, \ldots, n,
$$

for all real linear combinations of the elements $g_{1}, \ldots, g_{n}$. Then there exist $n$ real linear combinations

$$
G_{j}=\sum_{i=1}^{n} c_{j i} g_{i}, \quad H_{j}=\sum_{i=1}^{n} d_{j i} h_{i}, \quad c_{j i}, d_{j i} \in \mathbb{R}, \quad i, j=1, \ldots, n,
$$

such that

$$
\sum_{i=1}^{n} h_{i} \cdot g_{i}=\sum_{i=1}^{n} H_{i} \cdot G_{i}
$$

and that the valuations $v\left(H_{1}\right), \ldots, v\left(H_{n}\right)$ are pairwise distinct; then, of course, the valuations $v\left(H_{1} \cdot G_{1}\right), \ldots, v\left(H_{n} \cdot G_{n}\right)$ are pairwise distinct.

Proof. One can proceed with induction with respect to $n$. We show the case $n=2$. The general case is similar, the details being left to the reader. If $v\left(h_{1}\right) \neq v\left(h_{1}\right)$, we are done. Otherwise there are real numbers $d_{1}, d_{2} \neq 0$ such that

$$
v\left(d_{1} h_{1}-d_{2} h_{2}\right)>v\left(h_{1}\right)=v\left(h_{2}\right),
$$

and then

$$
\begin{aligned}
& h_{1} \cdot g_{1}+h_{2} \cdot g_{2}=d_{1} h_{1} \cdot d_{1}^{-1} g_{1}+d_{2} h_{2} \cdot d_{2}^{-1} g_{2}= \\
& =d_{1} h_{1} \cdot\left(d_{1}^{-1} g_{1}+d_{2}^{-1} g_{2}\right)+\left(d_{2} h_{2}-d_{1} h_{1}\right) \cdot d_{2}^{-1} g_{2} .
\end{aligned}
$$


Putting

$$
H_{1}:=d_{1} h_{1}, H_{2}:=d_{2} h_{2}-d_{1} h_{1}, G_{1}:=d_{1}^{-1} g_{1}+d_{2}^{-1} g_{2}, G_{2}:=d_{2}^{-1} g_{2},
$$

we get the required result.

Applying Lemma 4.3 to the elements

$$
h_{\alpha}=\varepsilon^{\alpha} \quad \text { and } \quad g_{\alpha}=f_{\alpha}\left(\mu, \delta_{1} / \mu, \tilde{\delta}\right), \quad \alpha \in \mathbb{N}^{p},
$$

we shall recursively define an increasing sequence of positive integers $\left(N_{k}\right)$ and three sequences of infinitesimals $\left(f_{k}\right),\left(\phi_{k}\right)$ and $\left(\psi_{k}\right), k \in \mathbb{N}$. Initially, let $\gamma \in G_{1}^{+}, N_{0}$ be any positive integer and

$$
A_{0}:=\left\{\alpha \in \mathbb{N}^{p}: v\left(\varepsilon^{\alpha}\right)<N_{0} \gamma\right\}, \quad n_{0}:=\sharp A_{0} .
$$

With the notation of Lemma 4.3, we get

$$
f_{0}:=\sum_{\alpha \in A_{0}} \varepsilon^{\alpha} \cdot g_{\alpha}=\sum_{i=1}^{n_{0}} H_{0, i} \cdot G_{0, i}
$$

put

$$
\psi_{0}:=0, \quad \varphi_{0}:=\sum\left\{H_{0, i} \cdot G_{0, i}: v\left(H_{i}\right)<N_{0} \gamma\right\} \quad \text { and } \quad \psi_{1}:=f_{0}-\varphi_{0} .
$$

Take $N_{1}$ so large that $v\left(H_{0, i}\right)<N_{1} \gamma$ for all $i=1, \ldots, n_{0}$. Let

$$
A_{1}:=\left\{\alpha \in \mathbb{N}^{p}: N_{0} \gamma \leq v\left(\varepsilon^{\alpha}\right)<N_{1} \gamma\right\}
$$

and $n_{1}$ be the sum of $\sharp A_{1}$ and the number of the summands of $\psi_{1}$. Again, with the notation of Lemma 4.3, we get

$$
f_{1}:=\sum_{\alpha \in A_{1} \backslash A_{0}} \varepsilon^{\alpha} \cdot g_{\alpha}+\psi_{1}=\sum_{i=1}^{n_{1}} H_{1, i} \cdot G_{1, i} ;
$$

clearly, $v\left(H_{1, i}\right) \geq N_{0} \gamma$ for $i=1, \ldots, n_{1}$; put

$$
\varphi_{1}:=\sum_{i}\left\{H_{1, i} \cdot G_{1, i}: v\left(H_{1, i}\right)<N_{1} \gamma\right\} \quad \text { and } \quad \psi_{2}:=f_{1}-\varphi_{1} .
$$

We continue this process recursively. By construction, each $\varphi_{k}$ is a finite sum of the form

$$
\varphi_{k}:=\sum_{i} H_{k, i} \cdot G_{k, i}, \quad \text { where } N_{k-1} \gamma \leq v\left(H_{k, i}\right)<N_{k} \gamma \text { for all } i,
$$

and the values $v\left(H_{k, i}\right)$ are pairwise distinct. It is easy to check that

$$
\nu=\sum_{\alpha \in \mathbb{N}^{p}} \varepsilon^{\alpha} \cdot g_{\alpha}=\sum_{k=0}^{\infty} f_{k}=\sum_{k=0}^{\infty} \varphi_{k} .
$$

We encounter two possibilities: either $G_{k, i} \in\langle\delta\rangle$ for each $k \in \mathbb{N}$ and every $i$, or there is a $k \in \mathbb{N}$ such that $G_{k, i} \notin\langle\delta\rangle$ for some $i$. We first show 
that the former one leads to a contradiction. Indeed, for each $k \in \mathbb{N}, G_{k, i}$ are of the form

$$
G_{k, i}=G_{k, i}\left(\mu, \delta_{1} / \mu, \tilde{\delta}\right), \quad i=1, \ldots, n_{k},
$$

where $G_{k, i}(u, v, \tilde{x})$ are functions $\mathcal{Q}$-analytic in a common neighbourhood of zero.

Since the infinitesimals $\mu, \delta$ are analytically independent, the tantamount conditions

$$
G_{k, i}\left(\mu, \delta_{1} / \mu, \tilde{\delta}\right) \in\langle\delta\rangle \quad \text { or } \quad G_{k, i}\left(\mu, \delta_{1} / \mu, \tilde{\delta}\right)=\tau(\delta)
$$

for an $\mathcal{L}$-term $\tau(x)$, hold iff the $\mathcal{L}$-term

$$
G_{k, i}\left(u, x_{1} / u, \tilde{x}\right)-\tau(x)
$$

vanishes on an open special cube containing the infinitesimals $\mu, \delta$. Then the partial derivative

$$
\partial / \partial u G_{k, i}\left(u, x_{1} / u, \tilde{x}\right)
$$

vanishes on that special cube and, in particular,

$$
\partial / \partial u G_{k, i}\left(\mu, \delta_{1} / \mu, \tilde{\delta}\right)=0 .
$$

Therefore, the former possibility implies that the partial derivative

$$
\partial / \partial u f\left(u, x_{1} / u, \tilde{x}, y\right)
$$

would vanish for $u=\mu, x=\delta$ and $y=\varepsilon$. Again, this partial derivative would vanish on a special cube containing the infinitesimals $\mu, \delta$ and $\varepsilon$, and thus it would vanish identically, by the identity principle for quasianalytic functions. As before, when considering Case A, we deduce that the function

$$
f\left(u, x_{1} / u, \tilde{x}, y\right)
$$

would coincide with a function $g(x, y) \mathcal{Q}$-analytic at zero. Hence

$$
\nu=g(\delta, \varepsilon) \in\langle\delta, \varepsilon\rangle=\langle\lambda\rangle,
$$

contrary to the assumption of $\left(\mathbf{I}_{\mathbf{m}}\right)$.

In this fashion, we may assume that the latter possibility holds. Obviously, we can write down the infinitesimal $\nu$ as below

$$
\nu=\sum_{k=0}^{\infty} \varphi_{k}=\sum_{j=0}^{\infty} H_{j} \cdot G_{j},
$$

where the values $v\left(H_{j}\right), j \in \mathbb{N}$, are pairwise distinct and for any $\gamma \in G_{1}$ there are only finitely many $j$ for which $v\left(H_{j}\right)<\gamma$. Under the circumstances, the set

$$
J:=\left\{j \in \mathbb{N}: G_{j} \notin\langle\delta\rangle\right\} \neq \emptyset
$$


is non-empty. There is, of course, a unique $j_{0} \in J$ such that

$$
v\left(H_{j_{0}}\right)=\min \left\{v\left(H_{j}\right): j \in J\right\} .
$$

Then

$$
I:=\left\{j \in \mathbb{N}: v\left(H_{i}\right)<v\left(H_{i_{0}}\right)\right\}
$$

is a finite subset of $\mathbb{N}$.

Now, it follows from the induction hypothesis $\left(\mathbf{I}_{\mathbf{m}-\mathbf{1}}\right)$ that there is an element $\tau(\delta) \in\langle\delta\rangle$ such that

$$
v\left(G_{j_{0}}-\tau(\delta)\right) \notin \Gamma_{\langle\delta\rangle} .
$$

Then

$$
\Lambda:=H_{j_{0}} \cdot \tau(\delta)+\sum_{j \in I} H_{j} \cdot G_{j} \in\langle\delta, \varepsilon\rangle=\langle\lambda\rangle,
$$

because $H_{j} \in\langle\varepsilon\rangle$ and $G_{j} \in\langle\delta\rangle$ for all $j \in I$. It is not difficult to check that

$$
v(\nu-\Lambda)=v\left(H_{j_{0}}\right)+v\left(G_{j_{0}}-\tau(\delta)\right) \notin \Gamma_{\langle\delta, \varepsilon\rangle}=\Gamma_{\langle\lambda\rangle} .
$$

This means that $\nu$ is active over the infinitesimals $\lambda$, which completes the proof of the theorem on an active infinitesimal $\left(\mathbf{I}_{\mathbf{m}}\right)$.

\section{QUANTIFIER ELIMINATION AND DESCRIPTION OF DEFINABLE FUNCTIONS BY TERMS}

In this section we are going to develop an approach to quantifier elimination and description of definable functions by terms in the language augmented by the names of rational powers, which is much shorter and more natural than the one in [20]. Observe first that one can introduce a welldefined notion of the dimension of sets defined piecewise by $\mathcal{L}$-terms. Indeed, every such set $E$ is a finite union of special cubes $S_{i}$ (op. cit., Theorem 2.1 and Corollary 2.3), and one can put

$$
\operatorname{dim} E:=\max \operatorname{dim} S_{i}
$$

It is easy to check that the dimension of a set $E$ does not depend on the decomposition into special cubes $S_{i}$.

By virtue of the exchange property for $\mathcal{L}$-terms from Section $3,\left(\mathbf{I V}_{\mathbf{m}}\right)$, $m \in \mathbb{N}$, (op. cit., Corollary 4.9), we have at our disposal a well-founded concept of rank for the substructures of a given model $\mathcal{R}$ of the universal diagram $T$. This allows us to establish the following result, which is a generalization of op. cit., Proposition 5.4. 
Proposition 5.1. Consider a map $f: \mathbb{R}^{d} \longrightarrow \mathbb{R}^{m}$ given piecewise by $\mathcal{L}$ terms and such that for every special cube $S \subset \mathbb{R}^{m}$ or, equivalently, for every subset $E$ of $\mathbb{R}^{m}$ given piecewise by $\mathcal{L}$-terms, we have

$$
\operatorname{dim} f^{-1}(S) \leq \operatorname{dim} S \text { or } \operatorname{dim} f^{-1}(E) \leq \operatorname{dim} E .
$$

Then $f$ admits a section given piecewise by $\mathcal{L}$-terms, i.e. there is a function $\xi: \mathbb{R}^{m} \longrightarrow \mathbb{R}^{d}$ given piecewise by $\mathcal{L}$-terms such that $f(\xi(y))=y$ for every point $y \in \mathbb{R}^{m}$.

Proof. We may, of course, assume that $f:(0,1)^{d} \longrightarrow(0,1)^{m}$. We first show that there exists a family $\left(t_{\iota}(y)\right)_{\iota \in I}$ of $\mathcal{L}$-terms, $t_{\iota}(y)=\left(t_{\iota, 1}(y), \ldots, t_{\iota, d}(y)\right)$, such that the infinite disjunction

$$
\bigvee_{\iota \in I}\left[\left(b=f(a) \wedge a \in(0,1)^{d}\right) \Longrightarrow b=f\left(t_{\iota}(b)\right)\right]
$$

holds for any tuples $a \in(0,1)^{d}$ and $b \in(0,1)^{m}$ in an arbitrary model $\mathcal{R}$ of the theory $T$. So take any elements $a \in(0,1)^{d}$ and $b \in(0,1)^{m}$ for which $b=\varphi(a)$. We may, of course, confine our analysis to the case where $a=\lambda$ and $b=\mu$ are infinitesimals. Let $k:=\operatorname{rk}\langle\lambda\rangle$; then the infinitesimals $\lambda$ lie on a special cube of dimension $k$, but lie on no special cube of dimension $<k$. Obviously,

$$
\langle\mu\rangle \subset\langle\lambda\rangle \text { and } \operatorname{rk}\langle\mu\rangle \leq \operatorname{rk}\langle\lambda\rangle .
$$

Were $\operatorname{rk}\langle\mu\rangle<k=\operatorname{rk}\langle\lambda\rangle$, then the infinitesimals $\mu$ would lie on a special cube $S$ of dimension $<k$, and thus it follows from the assumption that the infinitesimals $\lambda$ would lie on the set $f^{-1}(S)$ of dimension $<k$, which is impossible. Consequently,

$$
\operatorname{rk}\langle\mu\rangle=\operatorname{rk}\langle\lambda\rangle \quad \text { and }\langle\mu\rangle=\langle\lambda\rangle
$$

the last equality follows from the suitable property of rank operation (op. cit., Section 5), which is formulated in Section 3, assertion $\left(\mathbf{V}_{\mathbf{m}}\right), m \in \mathbb{N}$. Therefore the infinitesimals $\lambda$ can be expressed by $\mathcal{L}$-terms taken on the infinitesimals $\mu$, and the assertion follows.

Now, through model-theoretic compactness, one can find a finite set $\iota_{1}, \ldots, \iota_{n} \in I$ of indices for which the finite disjunction

$$
\bigvee_{k=1}^{n}\left[\left(b=f(a) \wedge a \in(0,1)^{d}\right) \Longrightarrow b=f\left(t_{\iota_{k}}(b)\right)\right]
$$

holds for any tuples $a$ and $b$ in an arbitrary model $\mathcal{R}$ of the theory $T$. In particular, this finite disjunction is satisfied in the standard model $\mathbb{R}_{\mathcal{Q}}$, concluding the proof of the proposition. 
Remark 5.2. The assumption of Proposition 5.1 is satisfied by every function $f$ given piecewise by $\mathcal{L}$-terms which is an immersion. More generally, consider a decomposition of $\mathbb{R}^{d}$ into finitely many leaves (i.e. $\mathcal{C}^{1}$ submanifolds) $F_{j}$ given piecewise by $\mathcal{L}$-terms, and a function $f$ given piecewise by $\mathcal{L}$-terms whose restriction to each $F_{j}$ is an immersion. Then $f$ satisfies that assumption too.

Corollary 5.3. Under the assumptions of Proposition 5.1, the image $f\left(\mathbb{R}^{d}\right)$ is given piecewise by $\mathcal{L}$-terms.

Proof. Indeed, suppose the section $\xi$ is given by a finite number of $\mathcal{L}$-terms

$$
\tau_{i}(y)=\left(\tau_{i 1}(y), \ldots, \tau_{i d}(y)\right), \quad i=1, \ldots, s, \quad y=\left(y_{1}, \ldots, y_{m}\right),
$$

i.e. $x=\xi(y)$ iff $\bigvee_{i=1}^{s} x=\tau_{i}(y)$. Then

$$
y \in f\left(\mathbb{R}^{d}\right) \Leftrightarrow \bigvee_{i=1}^{s} f\left(\tau_{i}(y)\right)=y,
$$

and thus the image $f\left(\mathbb{R}^{d}\right)$ is given by $\mathcal{L}$-terms $f\left(\tau_{i}(y)\right)-y, i=1, \ldots, s$, as desired.

By an immersion cube $C \subset \mathbb{R}^{m}$ we mean the image $\varphi\left((0,1)^{d}\right.$ where $\varphi$ is a $\mathcal{Q}$-map in a neighbourhood of the compact cube $[0,1]^{d}$, whose restriction to $(0,1)^{d}$ is an immersion. As demonstrated in our paper [19], the theorem on decomposition into special cubes along with the technique of fiber cutting make it possible to decompose every bounded $\mathcal{Q}$-subanalytic set into finitely many immersion cubes (op. cit., Corollary 1). This, in turn, and Corollary 5.3, immediately yield quantifier elimination for the expansion $\mathbb{R}_{\mathcal{Q}}$ of the real field with restricted quasianalytic functions in the language $\mathcal{L}$ augmented by the names of rational powers (cf. [20], Theorem 5.8):

Theorem 5.4. (Quantifier Elimination) Every set definable in the structure $\mathbb{R}_{\mathcal{Q}}$ is given piecewise by a finite number of $\mathcal{L}$-terms.

A fortiori, the structure $\mathbb{R}_{\mathcal{Q}}$ is model complete. Let us mention that one can apply decomposition into immersion cubes (cf. [19]) to prove that $\mathbb{R}_{\mathcal{Q}}$ is a polynomially bounded, o-minimal structure which admits $\mathcal{Q}$-analytic cell decomposition (being established in [27]); the last result requires an induction procedure (cf. [19]).

We now wish to turn to the problem of description of definable function by $\mathcal{L}$-terms.

Theorem 5.5. Each definable function $f: \mathbb{R}^{m} \longrightarrow \mathbb{R}$ is piecewise given by a finite number of $\mathcal{L}$-terms. 
Proof. Indeed, consider the graph $F \subset \mathbb{R}^{m+1}=\mathbb{R}_{x}^{m} \times \mathbb{R}_{y}$ of the function $f$ and denote by

$$
p: F \longrightarrow \mathbb{R}_{x}^{m} \text { and } q: F \longrightarrow \mathbb{R}_{y}
$$

the canonical projections. Via cell decomposition (of class $\mathcal{C}^{1}$ ), the graph $F$ can be partitioned into finitely many cells $C_{i}$ defined by $\mathcal{L}$-terms such that the restriction of $p$ to each cell $C_{i}$ is an immersion and, in fact, a diffeomorphism onto the image $p\left(C_{i}\right)$. It follows from Proposition 5.1 (cf. Remark 5.2) that each inverse

$$
p^{-1}: p\left(C_{i}\right) \longrightarrow C_{i}, \quad i=1, \ldots, s,
$$

is given piecewise by finitely many $\mathcal{L}$-terms, and thus so is the restriction of $f=q \circ p^{-1}$ to each set $p\left(C_{i}\right)$. This completes the proof.

We immediately obtain the following three corollaries.

Corollary 5.6. The structure $\mathbb{R}_{\mathcal{Q}}$ admits cell decompositions defined piecewise by $\mathcal{L}$-terms, and hence Skolem functions (of choice) given piecewise by $\mathcal{L}$-terms.

Corollary 5.7. The structure $\mathbb{R}_{\mathcal{Q}}$ is universally axiomatizable. Hence its universal diagram $T$ admits quantifier elimination (in the language $\mathcal{L}$ ) and $\mathbb{R}_{\mathcal{Q}}$ can be embedded as a prime model into each model of $T$. Consequently, in every model of $T$, each definable function is defined piecewise by a finite number of $\mathcal{L}$-terms.

Corollary 5.8. (Valuation Property for Definable Functions) Consider a simple (with respect to definable closure) extension $\mathcal{R} \subset \mathcal{R}\langle a\rangle$ of substructures in a fixed model of the theory $T$. Then we have the following dichotomy:

$$
\text { either } \quad \operatorname{dim} \Gamma_{\mathcal{R}\langle a\rangle}=\operatorname{dim} \Gamma_{\mathcal{R}} \quad \text { or } \quad \operatorname{dim} \Gamma_{\mathcal{R}\langle a\rangle}=\operatorname{dim} \Gamma_{\mathcal{R}}+1 .
$$

In the latter case, one can find an element $r \in \mathcal{R}$ such that

$$
v(a-r) \notin \Gamma_{\mathcal{R}} \quad \text { and } \quad \Gamma_{\mathcal{R}\langle a\rangle}=\Gamma_{\mathcal{R}} \oplus \mathbb{Q} \cdot v(a-r) .
$$

Remark 5.9. For the valuation property in the case of general, polynomially bounded, o-minimal structures and its connection with the preparation theorem in the sense of Parusiński-Lion-Rolin, see [7, 8] and also [18].

Similarly, we can immediately rephrase Theorem 2.1 for definable functions (cf. [21, Theorem 1]): 
Theorem 5.10. (On Rectilinearization of Definable Functions) If $f_{1}, \ldots, f_{s}$ : $\mathbb{R}^{m} \longrightarrow \mathbb{R}$ are definable functions and $K$ is a compact subset of $\mathbb{R}^{m}$, then there exists a finite collection of modifications

$$
\varphi_{i}:[-1,1]^{m} \longrightarrow R^{m}, \quad i=1, \ldots, p,
$$

such that

1) each $\varphi_{i}$ extends to a $\mathcal{Q}$-map in a neighbourhood of the cube $[-1,1]^{m}$, which is a composite of finitely many local blow-ups with smooth $\mathcal{Q}$-analytic centers and local power substitutions;

2) the union of the images $\varphi_{i}\left((-1,1)^{m}\right), i=1, \ldots, p$, is a neighbourhood of $K$.

3) for every bounded quadrant $Q_{j}, j=1, \ldots, 3^{m}$, the restriction to $Q_{j}$ of each function $f_{k} \circ \varphi_{i}, k=1, \ldots, s, i=1, \ldots, p$, either vanishes or is a normal crossing or a reciprocal normal crossing on $Q_{j}$.

Further, the following two results concerning rectilinearization of definable functions from our paper [21] can be repeated verbatim in the quasianalytic settings:

Theorem 5.11. (On Rectilinearization of a Definable Function) Let $U \subset$ $\mathbb{R}^{m}$ be a bounded open subset and $f: U \longrightarrow \mathbb{R}$ be a definable function. Then there exists a finite collection of modifications

$$
\varphi_{i}:[-1,1]^{m} \longrightarrow \mathbb{R}^{m}, \quad i=1, \ldots, p,
$$

such that

1) each $\varphi_{i}$ extends to a $\mathcal{Q}$-map in a neighbourhood of the cube $[-1,1]^{m}$, which is a composite of finitely many local blow-ups with smooth $\mathcal{Q}$-analytic centers and local power substitutions;

2) each set $\varphi_{i}^{-1}(U)$ is a finite union of bounded quadrants in $\mathbb{R}^{m}$;

3) each set $\varphi_{i}^{-1}(\partial U)$ is a finite union of bounded closed quadrants in $\mathbb{R}^{m}$ of dimension $m-1$;

4) $U$ is the union of the images $\varphi_{i}(\operatorname{Int}(Q))$ with $Q$ ranging over the bounded quadrants contained in $\varphi_{i}^{-1}(U), i=1, \ldots, p$;

5) for every bounded quadrant $Q$, the restriction to $Q$ of each function $f \circ \varphi_{i}$ either vanishes or is a normal crossing or a reciprocal normal crossing on $Q$, unless $\varphi_{i}^{-1}(U) \cap Q=\emptyset$.

Remark 5.12. One can formulate Theorem 5.11 for several definable functions $f_{1}, \ldots, f_{s}$.

It follows from points 1) and 2) that every bounded quadrant of dimension $<m$ contained in $\varphi_{i}^{-1}(U)$ is adjacent to a bounded quadrant of 
dimension $m$ (a bounded orthant) contained in $\varphi_{i}^{-1}(U)$. Hence

$$
\varphi_{i}^{-1}(\bar{U})=\overline{\varphi_{i}^{-1}(U)}
$$

and therefore point 4) implies that $\bar{U}$ is the union of the images $\varphi_{i}(\bar{Q})$ of the closures of those bounded quadrants of dimension $m$ (bounded orthants) $Q$ for which $\varphi_{i}(Q) \subset U, i=1, \ldots, p$.

For a bounded orthant $Q$ contained in $\varphi_{i}^{-1}(U)$, denote by $\operatorname{dom}_{i}(Q)$ the union of $Q$ and all those bounded quadrants that are adjacent to $Q$ and disjoint with $\varphi_{i}^{-1}(\partial U)$; it is, of course, an open subset of the closure $\bar{Q}$. Moreover, the open subset $\varphi_{i}^{-1}(U)$ of the cube $[-1,1]^{m}$ coincides with the union of $\operatorname{dom}_{i}(Q)$, where $Q$ range over the bounded orthants that are contained in $\varphi_{i}^{-1}(U)$, and with the union of those bounded quadrants that are contained in $\varphi_{i}^{-1}(U)$. Consequently, the union of the images $\varphi_{i}(\operatorname{Int}(Q))$, where $Q$ range over the bounded quadrants that are contained in $\varphi_{i}^{-1}(U)$, coincides with the union of the images

$$
\varphi_{i}\left(\operatorname{dom}_{i}(Q) \cap(-1,1)^{m}\right),
$$

where $Q$ range over the bounded orthants $Q$ that are contained in $\varphi_{i}^{-1}(U)$. Thus we get

Corollary 5.13. (On Rectilinearization of a Continuous Definable Function) Let $U$ be a bounded open subset in $\mathbb{R}^{m}$ and $f: U \longrightarrow \mathbb{R}$ be a continuous definable function. Then there exists a finite collection of modifications

$$
\varphi_{i}:[-1,1]^{m} \longrightarrow \mathbb{R}^{m}, \quad i=1, \ldots, p,
$$

such that

1) each $\varphi_{i}$ extends to a $\mathcal{Q}$-map in a neighbourhood of the cube $[-1,1]^{m}$, which is a composite of finitely many local blow-ups with smooth $\mathcal{Q}$-analytic centers and local power substitutions;

2) each set $\varphi_{i}^{-1}(U)$ is a finite union of bounded quadrants in $\mathbb{R}^{m}$;

3) each set $\varphi_{i}^{-1}(\partial U)$ is a finite union of bounded closed quadrants in $\mathbb{R}^{m}$ of dimension $m-1$;

4) $U$ is the union of the images $\varphi_{i}\left(\operatorname{dom}_{i}(Q) \cap(-1,1)^{m}\right)$ with $Q$ ranging over the bounded orthants $Q$ contained in $\varphi_{i}^{-1}(U), i=1, \ldots, p$;

5) for every bounded orthant $Q$, the restriction to $\operatorname{dom}_{i}(Q)$ of each function $f \circ \varphi_{i}$ either vanishes or is a normal crossing or a reciprocal normal crossing on $Q$, unless $\varphi_{i}^{-1}(U) \cap Q=\emptyset$.

Remark 5.14. Observe that in the foregoing rectilinearization results, if the functions $f_{1}, \ldots, f_{s}$ are given piecewise by terms in the language of restricted 
$\mathcal{Q}$-analytic functions augmented merely by the reciprocal function $1 / x$, then one can require that the modifications $\varphi_{i}, i=1, \ldots, p$, be composite of finitely many local blow-ups with smooth $\mathcal{Q}$-analytic centers.

\section{Power substitution for Denjoy-Carleman classes}

Consider an increasing sequence $M=\left(M_{n}\right)$ of real numbers with $M_{0}=1$. Let $I$ be an interval (open or closed) contained in $\mathbb{R}$. By $\mathcal{Q}(I, M)$ we denote the class of functions on $I$ that satisfy the following growth condition for their derivatives:

$$
\left|\partial^{|\alpha|} f / \partial x^{\alpha}(x)\right| \leq C \cdot R^{|\alpha|} \cdot|\alpha| ! \cdot M_{|\alpha|} \quad \text { for all } \quad x \in I, \alpha \in \mathbb{N}^{n},
$$

with some constants $C, R>0$.

The main purpose of this section is to prove the following

Theorem 6.1. Let $p>1$ be an integer and I the interval $[0,1]$ or $[-1,1]$ according as $p$ is even or odd. Consider power substitution $x=\xi^{p}$, which is a bijection of $I$ onto itself. Let $f: I \longrightarrow \mathbb{R}$ be a smooth function. If

$$
F(\xi):=(f \circ \varphi)(\xi)=f\left(\xi^{p}\right) \in \mathcal{Q}(I, M),
$$

then $f(x) \in \mathcal{Q}\left(I, M^{(p)}\right)$, where the sequence $M^{(p)}$ is defined by putting $M_{n}^{(p)}:=M_{p n}$.

Equivalently, in terms of the corresponding sequences $M^{\prime}$ (see the Introduction), the function $f$ belongs to the class determined by the sequence $M_{n}^{\prime(p)}:=1 / n^{(p-1) n} \cdot M_{p n}^{\prime}, n \in \mathbb{N}$.

Remark 6.2. Using a function constructed by Bang, we shall show at the end of this section that, in the case where $p=2$ and the sequence $M^{\prime}$ is log-convex, $\mathcal{Q}\left(I, M^{(2)}\right)$ is the smallest Denjoy-Carleman class containing all those functions $f(x)$.

Remark 6.3. The case $p=2$ of Theorem 6.1 may be related to the following problem, which was investigated by Mandelbrojt [15, 16] and solved completely by Lalaguë [12, Chap. III].

Consider a smooth function $f(x)$ on the interval $[-1,1]$ and suppose that $F(\xi):=f(\cos \xi)$ belongs to a class $\mathcal{Q}(\mathbb{R}, M)$. To which class on the interval $[-1,1]$ does $f$ belong?

Proof. Before establishing Theorem 6.1, we state two lemmas below. 
Lemma 6.4. Consider the Taylor expansions

$$
\sum_{i=1}^{\infty} \frac{x^{i}}{i}=-\log (1-x) \quad \text { and } \quad\left(\sum_{i=1}^{\infty} \frac{x^{i}}{i}\right)^{k}=\sum_{n=1}^{\infty} c_{k, n} x^{n} .
$$

Then we have the estimate

$$
c_{k, n} \leq(2 e)^{n} \cdot \frac{k !}{n^{k}} \quad \text { for all } \quad k, n \in \mathbb{N} .
$$

Proof. Indeed, it is easy to verify the estimate:

$$
|\log (1-z)| \leq\left|\log 2+\frac{\pi}{6} \sqrt{-1}\right| \leq 1 \quad \text { for all } \quad z \in \mathbb{C},|z| \leq 1 / 2 .
$$

By Cauchy's inequalities, we thus get $\left|c_{k, n}\right| \leq 2^{n}$. Since $e^{n}>n^{k} / k$ ! for all $n, k \in \mathbb{N}$, we have

$$
c_{k, n} \leq 2^{n}<2^{n} \cdot e^{n} \cdot \frac{k !}{n^{k}}=(2 e)^{n} \cdot \frac{k !}{n^{k}},
$$

as asserted.

As an immediate consequence, we obtain

\section{Corollary 6.5.}

$$
\sum_{i_{1}+\ldots+i_{k}=n} \frac{1}{i_{1}} \cdot \ldots \cdot \frac{1}{i_{k}}=c_{k, n} \leq(2 e)^{n} \cdot \frac{k !}{n^{k}} \quad \text { for all } \quad k, n \in \mathbb{N} .
$$

Lemma 6.6. Let $p, k \in \mathbb{N}$ with $p>1, k \geq 1$, and

$$
\alpha_{k}(X, x):=\frac{1}{k !}\left(X^{1 / p}-x^{1 / p}\right)^{k} \quad \text { for } \quad X, x>0,
$$

where $\alpha_{k}$ is regarded as a function in one variable $X$ and parameter $x$. Then we have the estimate

$$
\left|\alpha_{k}^{(n)}(x, x)\right| \leq(2 e)^{n} \cdot n^{n-k} \cdot x^{-\frac{p n-k}{p}} \quad \text { for all } n, k \in \mathbb{N}, \quad x>0 .
$$

Proof. Consider first the case $k=1, \alpha_{1}(X, x)=X^{1 / p}-x^{1 / p}$. Then

$$
\alpha_{1}^{(n)}(x, x)= \pm \frac{(p-1)(2 p-1) \cdot \ldots \cdot((n-1) p-1)}{p^{n}} \cdot x^{-\frac{p n-1}{p}},
$$

whence

$$
\left|\alpha_{1}^{(n)}(x, x)\right| \leq(n-1) ! \cdot x^{-\frac{p n-1}{p}} \leq n^{n-1} \cdot x^{-\frac{p n-1}{p}},
$$

as asserted. The Taylor expansion of $\alpha_{1}(X, x)$ at $X=x$ is

$$
\alpha_{1}(X, x)=\sum_{i=1}^{\infty} a_{i} \cdot x^{-\frac{p i-1}{p}}(X-x)^{i}
$$

where

$$
a_{i}:= \pm \frac{1}{i !} \cdot \frac{(p-1)(2 p-1) \cdot \ldots \cdot((i-1) p-1)}{p^{i}}
$$


obviously, $\left|a_{i}\right| \leq(i-1) ! / i !=1 / i$. We thus get

$$
\alpha_{k}(X, x)=\frac{1}{k !}\left(\sum_{i=1}^{\infty} a_{i} \cdot x^{-\frac{p i-1}{p}}(X-x)^{i}\right)^{k}=\sum_{j=1}^{\infty} b_{j} \cdot x^{-\frac{p j-k}{p}}(X-x)^{j},
$$

where

$$
b_{j}:=\frac{1}{k !} \sum_{i_{1}+\ldots+i_{k}=j} a_{i_{1}} \cdot \ldots \cdot a_{i_{k}} .
$$

Then

$$
\begin{gathered}
\left|b_{n}\right| \leq \frac{1}{k !} \sum_{i_{1}+\ldots+i_{k}=n}\left|a_{i_{1}}\right| \cdot \ldots \cdot\left|a_{i_{k}}\right| \leq \frac{1}{k !} \sum_{i_{1}+\ldots+i_{k}=n} \frac{1}{i_{1}} \cdot \ldots \cdot \frac{1}{i_{k}}= \\
=\frac{c_{k, n}}{k !} \leq \frac{(2 e)^{n}}{n^{k}} \quad \text { for all } n, k \in \mathbb{N} ;
\end{gathered}
$$

the last inequality follows from Corollary 6.5. Hence

$$
\left|\alpha_{k}^{(n)}(x, x)\right| \leq n ! \cdot\left|b_{n}\right| \cdot x^{-\frac{p n-k}{p}} \leq(2 e)^{n} \cdot \frac{n !}{n^{k}} \cdot x^{-\frac{p n-k}{p}} \leq(2 e)^{n} \cdot n^{n-k} \cdot x^{-\frac{p n-k}{p}}
$$

for all $n, k \in \mathbb{N}, x>0$, as asserted.

Now we can readily pass to the proof of Theorem 6.1. We shall work with estimates corresponding to the sequence $M^{\prime}$. So suppose that

$$
\left|F^{(n)}(\xi)\right| \leq A^{n} M_{n}^{\prime}
$$

for all $n \in \mathbb{N}, \xi \in I$ and some constant $A>0$. We are going to estimate the growth of the $n$-th derivative $f^{(n)}$. Fix $n \in \mathbb{N}$ and put:

$$
\begin{gathered}
p_{n}(x):=T_{0}^{n} f(x)=\sum_{k=0}^{n-1} f^{k}(0) \frac{x^{k}}{k !}, \quad r_{n}(x):=f(x)-p_{n}(x), \\
P_{n}(\xi):=p_{n}\left(\xi^{p}\right) \quad \text { and } \quad R_{n}(\xi):=r_{n}\left(\xi^{p}\right) .
\end{gathered}
$$

Obviously,

$$
P_{n}^{(p n)} \equiv 0 \quad \text { and } \quad R_{n}^{(p n)} \equiv F^{(p n)} .
$$

From the Taylor formula, we therefore obtain the estimate

$$
\left|R_{n}^{(p n)}(\xi)\right| \leq A^{p n} M_{p n}^{\prime} \cdot \frac{\xi^{p n-k}}{(p n-k) !}
$$

for all $k<p n, \xi \in I$. We still need an elementary inequality

$$
\frac{1}{(p n-k) !} \leq \frac{e^{p n}}{n^{p n-k}} \quad \text { for all } \quad k<p n \text {. }
$$

For, it suffices to show that

$$
\frac{e^{p n-k}}{(p n-k)^{p n-k}} \leq \frac{e^{p n}}{n^{p n-k}}
$$


When $p n-k \geq n$ or, equivalently, $k \leq(p-1) n$, the last inequality is evident. Suppose thus that $p n-k<n$ or, equivalently, $k>(p-1) n$. This inequality is, of course, equivalent to

$$
\left(\frac{n}{p n-k}\right)^{p n-k} \leq e^{k}
$$

which holds as shown below:

$$
\begin{aligned}
& \left(\frac{n}{p n-k}\right)^{p n-k}=\left(1+\frac{k-(p-1) n}{p n-k}\right)^{p n-k}= \\
& =\left[\left(1+\frac{k-(p-1) n}{p n-k}\right)^{\frac{p n-k}{k-(p-1) n}}\right]^{k-(p-1) n}<e^{k} .
\end{aligned}
$$

Now, the foregoing estimate along with inequality (6.1) yield

$$
\left|R_{n}^{(p n)}(\xi)\right| \leq A^{p n} M_{p n}^{\prime} \cdot \frac{e^{p n}}{n^{p n-k}} \cdot \xi^{p n-k}
$$

Applying the formula for the derivatives of a composite function, we obtain

$$
r_{n}^{(n)}(x)=\sum_{k=1}^{n} R_{n}^{(k)}(\xi) \cdot \alpha_{k}^{(n)}(x, x) .
$$

Hence and by Lemma 6.6, we get

$$
\begin{gathered}
\left|f^{(n)}(x)\right|=\left|r_{n}^{(n)}(x)\right| \leq \sum_{k=1}^{n} A^{p n} M_{p n}^{\prime} \cdot \frac{e^{p n}}{n^{p n-k}} \cdot|\xi|^{p n-k} \cdot(2 e)^{n} n^{n-k} \cdot|\xi|^{-(p n-k)}= \\
=n \cdot(2 e)^{n} \cdot(e A)^{p n} \cdot \frac{M_{p n}^{\prime}}{n^{(p-1) n}},
\end{gathered}
$$

which completes the proof of Theorem 6.1.

Finally, we show that, when the sequence $M^{\prime}$ is log-convex, $\mathcal{Q}\left(I, M^{(2)}\right)$ is the smallest Denjoy-Carleman class containing all smooth functions $f(x)$ on the interval $I=[0,1]$ such that $F(\xi)=f\left(\xi^{2}\right) \in \mathcal{Q}(I, M)$. We make use of a classical function constructed by Bang [1], applied in his proof that the classes determined by log-convex sequences contain functions with sufficiently large derivatives (the result due to Cartan [4] and Mandelbrojt [5]; see also [30], Section 1, Theorem 1).

The logarithmic convexity of the sequence $M^{\prime}$ yields for every $j, k \in \mathbb{N}$ the inequality

$$
\left(\frac{1}{m_{k}}\right)^{k-j} \leq \frac{M_{j}^{\prime}}{M_{k}^{\prime}} \quad \text { where } \quad m_{k}:=\frac{M_{k+1}^{\prime}}{M_{k}^{\prime}}
$$

Consequently,

$$
F(\xi):=\sum_{k=0}^{\infty} \frac{M_{k}^{\prime}}{\left(2 m_{k}\right)^{k}} \cos \left(2 m_{k} \xi\right)
$$


is an even smooth function on $\mathbb{R}$ such that

$$
F(\xi) \in \mathcal{Q}(\mathbb{R}, M) \text { and } \quad\left|F^{(2 n)}(0)\right| \geq M_{2 n}^{\prime}
$$

for all $n \in \mathbb{N}$. Therefore $F(\xi)=f\left(\xi^{2}\right)$ for some smooth function $f$ on $\mathbb{R}$ (cf. [32]), and we get

$$
f^{(n)}(0)=\frac{n !}{(2 n) !} F^{(2 n)}(0) \quad \text { and } \quad\left|f^{(n)}(0)\right| \geq \frac{n ! M_{2 n}^{\prime}}{(2 n) !}
$$

which is the desired result.

We conclude this section with some examples, one of which (namely, for $k=2$ ) will be applied to the construction of our counterexample in the last section.

Example 6.7. Fix an integer $k \in \mathbb{N}, k \geq 1$, and put

$$
\log ^{(k)}:=\underbrace{\log \circ \ldots \circ \log }_{k \text { times }}, \quad \text { and } \quad e \uparrow \uparrow k:=(\underbrace{\exp \circ \ldots \circ \exp }_{k \text { times }})(1)
$$

let $n_{k}$ be the smallest integer greater than $e \uparrow \uparrow k$. Then the sequence

$$
\left(\log ^{(k)} n\right)^{n} \quad \text { for } \quad n \geq n_{k}
$$

is log-convex. Further, the shifted sequence:

$$
M=\left(M_{n}\right), \quad M_{n}:=\frac{1}{\left(\log ^{(k)} n_{k}\right)^{n_{k}}} \cdot\left(\log ^{(k)}\left(n_{k}+n\right)\right)^{\left(n_{k}+n\right)},
$$

determines a quasianalytic class closed under derivatives; the former follows from Cauchy's condensation criterion. It is easy to check that the sequences $M^{(p)}, p>1$, are quasianalytic when $k>1$, but are not quasianalytic when $k=1$.

\section{NON-EXTENDABILITY OF QUASIANALYTIC GERMS}

In this section we are concerned with a result by V. Thilliez [31] on the extension of quasianalytic function germs in one variable, recalled below. As before, consider two log-convex sequences $M$ and $N$ with $M_{0}=N_{0}=1$ such that $\mathcal{Q}_{1}(M) \subset \mathcal{Q}_{1}(N)$. Denote by $\mathcal{Q}_{1}(M)^{+}$the local ring of right-hand side germs at zero (i.e. germs of functions from $\mathcal{Q}([0, \varepsilon], M)$ for some $\varepsilon>0)$.

Theorem 7.1. If $\mathcal{Q}_{1}(N)$ is a quasianalytic local ring, then

$$
\mathcal{O}_{1} \varsubsetneqq \mathcal{Q}_{1}(M) \subset \mathcal{Q}_{1}(N) \Longrightarrow \mathcal{Q}_{1}(M)^{+} \backslash \mathcal{Q}_{1}(N) \neq \emptyset,
$$

i.e. there exist right-hand side germs from $\mathcal{Q}_{1}(M)^{+}$which do not extend to germs from $\mathcal{Q}_{1}(N)$. Here $\mathcal{O}_{1}$ stands for the local ring of analytic function germs in one variable at $0 \in \mathbb{R}$. 
Remark 7.2. Theorem 7.1 may be related to the research by M. Langenbruch [13] on the extension of ultradifferentiable functions in several variables, principally focused on the non-quasianalytic case, which seems to be more difficult in this context. His extension problem is, roughly speaking, as follows:

Given two compact convex subsets $K, K_{1}$ of $\mathbb{R}^{m}$ such that int $(K) \neq \emptyset$ or $K=\{0\}$ and $K \subset \operatorname{int}\left(K_{1}\right)$, characterize the sequences of positive numbers $M$ and $N$ such that every function from the class $\mathcal{Q}(K, M)$ extends to a function from $\mathcal{Q}\left(K_{1}, N\right)$.

M. Langenbruch applies, however, different methods and techniques in comparison with V. Thilliez. In particular, his approach is based on the theory of Fourier transform and plurisubharmonic functions.

On the other hand, Thilliez's approach relies on Grothendieck's version of the open mapping theorem (cf. [9], Chap. 4, Part 1, Theorem 2 or [17], Part IV, Chap. 24) and Runge approximation. It also enables a formulation of the non-extendability theorem for quasianalytic function germs on a compact convex subset $K \subset \mathbb{R}^{m}$ with $0 \in K$.

Nevertheless, in order to construct our counterexample in the next section, we need a refinement of Theorem 7.1, stated below. Thilliez's proof can be adapted mutatis mutandis. We shall outline it for the reader's convenience. Consider an increasing countable family $M^{[p]}, p \in \mathbb{N}$, of log-convex sequences, i.e.

$$
1=M_{0}^{[p]} \leq M_{1}^{[p]} \leq M_{2}^{[p]} \leq M_{3}^{[p]} \leq \ldots \quad \text { and } \quad M_{j}^{[p]} \leq M_{j}^{[q]}
$$

for all $j, p, q \in \mathbb{N}, p \leq q$. Then we receive an ascending sequence of local rings

$$
\mathcal{Q}_{1}\left(M^{[1]}\right) \subset \mathcal{Q}_{1}\left(M^{[2]}\right) \subset \mathcal{Q}_{1}\left(M^{[3]}\right) \subset \ldots
$$

such that $\mathcal{Q}_{1}\left(M^{[p]}\right)$ is dominated by $\mathcal{Q}_{1}\left(M^{(q)}\right)$ for all $p, q \in \mathbb{N}$ with $p \leq q$.

Theorem 7.3. If every local ring $\mathcal{Q}_{1}\left(M^{[p]}\right)$ is quasianalytic, then

$$
\mathcal{O}_{1} \varsubsetneqq \mathcal{Q}_{1}(M) \subset \bigcup_{p \in \mathbb{N}} \mathcal{Q}_{1}\left(M^{[p]}\right) \Longrightarrow \mathcal{Q}_{1}(M)^{+} \backslash \bigcup_{p \in \mathbb{N}} \mathcal{Q}_{1}\left(M^{[p]}\right) \neq \emptyset .
$$

Proof. We adopt the following notation. For a smooth function $f$ on an interval $I \subset \mathbb{R}$ and $r>0$, put

$$
\|f\|_{M, I, r}:=\sup \left\{\frac{\left|f^{(n)}(x)\right|}{r^{n} n ! M_{n}}: n \in \mathbb{N}, x \in I\right\} \text {. }
$$


For $k \in \mathbb{N}, k>0$, let $B_{k}(M)$ or $B_{k}(M)^{+}$, respectively, denote the Banach space with norm

$$
\|\cdot\|_{M,[-1 / k, 1 / k], k} \quad \text { or } \quad\|\cdot\|_{M,[0,1 / k], k}
$$

of those smooth functions on the interval $[-1 / k, 1 / k]$ or $[0,1 / k])$ such that

$$
\|f\|_{M,[-1 / k, 1 / k], k}<\infty \quad \text { or } \quad\|f\|_{M,[0,1 / k], k}<\infty, \text { respectively. }
$$

As the canonical embeddings

$$
B_{k}(M) \hookrightarrow B_{l}(M) \quad \text { and } \quad B_{k}(M)^{+} \hookrightarrow B_{l}(M)^{+}, \quad k, l \in \mathbb{N}, k \leq l,
$$

are compact linear operators (a consequence of Ascoli's theorem; cf. [10]), one can endow the local rings $\mathcal{Q}_{1}(M)$ and $\mathcal{Q}_{1}(M)^{+}$with the inductive topologies. Similarly, the countable union of local rings

$$
\bigcup_{p \in \mathbb{N}} \mathcal{Q}_{1}\left(M^{[p]}\right)
$$

is the inductive limit of the sequence $B_{k}\left(M^{(k)}\right), k \in \mathbb{N}, k>0$, of Banach algebras. Further, the local ring

$$
\bigcup_{p \in \mathbb{N}} \mathcal{Q}_{1}\left(M^{[p]}\right) \cap \mathcal{Q}_{1}(M)^{+}
$$

is the inductive limit of the sequence

$$
B_{k}\left(M^{(k)}\right) \cap B_{k}(M)^{+}, \quad k \in \mathbb{N}, k>0,
$$

of Banach algebras with norms

$$
\|f\|_{k}:=\max \left\{\|f\|_{M^{[k],[-1 / k, 1 / k], k}},\|f\|_{M,[0,1 / k], k}\right\} .
$$

Clearly, the restriction operator

$$
R: \bigcup_{p \in \mathbb{N}} \mathcal{Q}_{1}\left(M^{[p]}\right) \cap \mathcal{Q}_{1}(M)^{+} \longrightarrow \mathcal{Q}_{1}(M)^{+}
$$

is continuous and injective by quasianalyticity. We must show that $R$ is not surjective.

To proceed with reduction ad absurdum, suppose that $R$ is surjective. By Grothendieck's version of the open mapping theorem (cf. [9], Chap. 4, Part 1, Theorem 2 or [17], Part IV, Chap. 24), the operator $R$ is a homeomorphism onto the image. Further, by Grothendieck's factorization theorem (loc. cit.), for each $k \in \mathbb{N}$ there is an $l \in \mathbb{N}$ and a constant $C>0$ such that

$$
R\left(B_{l}\left(M^{(l)}\right)\right) \supset R\left(B_{l}\left(M^{(l)}\right) \cap B_{l}(M)^{+}\right) \supset B_{k}(M)^{+},
$$

and

$$
\left\|R^{-1} f\right\|_{M^{(l),[-1 / l, 1 / l], l}} \leq\left\|R^{-1} f\right\|_{l} \leq C\|f\|_{M,[0,1 / k], k}
$$


for all $f \in B_{k}(M)^{+}$. In particular, there is an $l \in \mathbb{N}$ and a constant $A>0$ such that

$$
R\left(B_{l}\left(M^{(l)}\right)\right) \supset B_{1}(M)^{+}
$$

and

$$
\left\|R^{-1} f\right\|_{M^{(l)},[-1 / l, 1 / l], l} \leq A\|f\|_{M,[0,1], 1}
$$

for all $f \in B_{1}(M)^{+}$. In particular,

$$
\left|P\left(-\frac{1}{l}\right)\right| \leq A\|P\|_{M,[0,1], 1}
$$

for every polynomial $P \in \mathbb{C}[x]$. Put

$$
W:=\left\{z \in \mathbb{C}: \operatorname{dist}(z,[0,1]) \leq \frac{1}{2 l}\right\}
$$

and

$$
B:=\sup \left\{\frac{(2 l)^{n}}{M_{n}}: n \in \mathbb{N}\right\}<\infty
$$

the last inequality holds because $\mathcal{O}_{1} \varsubsetneqq \mathcal{Q}_{1}(M)$ whence

$$
\sup \left\{\sqrt[n]{M_{n}}: n \in \mathbb{N}\right\}=\infty
$$

It follows from Cauchy's inequalities that

$$
\sup \left\{\left|P^{(n)}(x)\right|: x \in[0,1]\right\} \leq n !(2 l)^{n} \sup \{|P(x)|: x \in W\},
$$

and hence

$$
\|P\|_{M,[0,1], 1} \leq B \sup \{|P(x)|: x \in W\}
$$

Consequently,

$$
\left|P\left(-\frac{1}{l}\right)\right| \leq A B \sup \{|P(x)|: x \in W\}
$$

for every polynomial $P \in \mathbb{C}[x]$. But, by virtue of Runge approximation, there exists a sequence of polynomials $P_{\nu} \in \mathbb{C}[x]$ which converges uniformly to 0 on $W$, and to 1 for $x=-1 / l$. This contradicts the above estimate, and thus the theorem follows.

\section{Construction of a Counterexample}

We give a counterexample indicating that quasianalytic structures, unlike the classical structure $\mathbb{R}_{a n}$, may not admit quantifier elimination in the language augmented merely by the reciprocal function $1 / x$. The example we construct is a plane curve through $0 \in \mathbb{R}^{2}$ which is definable in the quasianalytic structure corresponding to the log-convex sequence

$$
M=\left(M_{n}\right), \quad M_{n}:=\frac{1}{(\log \log 3)^{3}} \cdot(\log \log (n+3))^{(n+3)} ;
$$


this sequence determines a quasianalytic class closed under derivatives (cf. example 6.7). By Theorem 7.3, we can take a function germ

$$
f \in \mathcal{Q}_{1}(M)^{+} \backslash \bigcup_{p \text { odd }} \mathcal{Q}_{1}\left(M^{(p)}\right)
$$

Let $V \subset \mathbb{R}^{2}$ be the graph of a representative of this germ in a right-hand side neighbourhood $[0, \varepsilon]$.

To proceed with reductio ad absurdum, suppose $V$ is given by a term in the language of restricted $\mathcal{Q}_{M}$-analytic functions augmented merely by the reciprocal function $1 / x$. Taking into account Remark 2.2 , we can thus deduce from Theorem 2.1 that there would exist a rectilinearization of this term by a finite sequence of blow-ups of the real plane at points. Consequently, the germ of $V$ at zero would be contained in the image $\varphi([-1,1])$, where

$$
\varphi=\left(\varphi_{1}, \varphi_{2}\right):[-1,1] \longrightarrow \mathbb{R}^{2}, \varphi(0)=0
$$

is a $\mathcal{Q}_{M}$-analytic homeomorphism. But then the order of $\varphi_{1}$ at zero must be odd, and thus the set $V$ would have a parametrization near zero of the form $\left(\xi^{p}, g(\xi)\right)$, where $p$ is an odd positive integer and $g$ is a $\mathcal{Q}_{M}$-function in the vicinity of zero. Hence and by Theorem 6.1, we would get

$$
f(x)=g\left(x^{1 / p}\right) \in \mathcal{Q}_{1}\left(M^{(p)}\right),
$$

which is a contradiction.

Remark 8.1. By virtue of Puiseux's theorem for definable functions (cf. [23], Section 2), the germ of every smooth function in one variable that is definable in the structure $\mathbb{R}_{\mathcal{Q}_{M}}$ belongs to $\mathcal{Q}_{1}\left(M^{(p)}\right)$ for some positive integer $p$. Therefore the structure under study will not admit quantifier elimination, even considered with the richer language of restricted definable quasianalytic functions augmented by the reciprocal function $1 / x$.

Remark 8.2. Also, our counterexample demonstrates that the classical theorem of Łojasiewicz [14] that every subanalytic set of dimension $\leq 1$ is semianalytic is no longer true in quasianalytic structures.

Acknowledgements. This research was partially supported by Research Project No. N N201 372336 from the Polish Ministry of Science and Higher Education. 


\section{REFERENCES}

[1] T. Bang, Om quasi-analytiske Funktioner, Thesis, University of Copenhagen, 1946.

[2] E. Bierstone, P.D. Milman, Canonical desingularization in characteristic zero by blowing up the maximum strata of a local invariant, Inventiones Math. 128 (1997), 207-302.

[3] —, - Resolution of singularities in Denjoy-Caleman classes, Selecta Math., New Ser. 10 (2004), 1-28.

[4] H. Cartan, Sur les classes de fomctions définies par des inégalités portant sur leurs dérivées successives, Actual. Sci. Ind. 867, Hermann, Paris, 1940.

[5] —, S. Mandelbrojt, Solution du problème d'équivalence des classes de fonctions indéfiniment dérivables, Acta Math. 72 (1940), 41-49.

[6] J. Denef, L. van den Dries, p-adic and real subanalytic sets, Ann. Math. 128 (1988), $79-138$.

[7] L. van den Dries, P. Speissegger, The field of reals with multisummable series and the exponential function, Proc. London Math. Soc. (3), 81 (2000), 513-565

[8] - - - O-minimal preparation theorems. In: Proc. Euro-Conference in Model Theory and its Applications, Ravello, Italy, 2002.

[9] A. Grothendieck, Topological Vector Spaces, Gordon \& Breach Sci. Publ., New York, London, Paris, 1973.

[10] H. Komatsu, Ultradistributions, I. Structure theorems and characterization, J. Fac. Sci. Univ. Tokyo, Sect. IA 20 (1973), 25-105.

[11] —, The implicit function theorem for ultradifferentiable mappings, Proc. Japan Acad. 55 (1979), 69-72.

[12] P. Lalaguë, Sur certaines classes de fonctions indéfiniment dérivables, Ann. Sci. École Norm. Sup. 72 (1955), 237-298.

[13] M. Langenbruch, Extension of ultradifferentiable functions, Manuscr. Math. 83 (1994), 123-143.

[14] S. Łojasiewicz, Ensembles semi-analytiques, Inst. Hautes Études Sci., Bures-surYvette, 1964.

[15] S. Mandelbrojt, Quelques nouvelles considerations sur les classes de fonctions dont les derivées sont bornes, C. R. Math. Acad. Sci. Paris 208 (1939), 1202.

[16] —, Séries adhérentes. Régularisation des suites. Applications, Coll. Borel, GauthierVillars, 1952.

[17] R. Meise, D. Vogt, Introduction to Functional Analysis, Oxford University Press, New York, 1997.

[18] K.J. Nowak, A proof of the valuation property and preparation theorem, Ann. Polon. Math. 92 (1) (2007), 75-85.

[19] —, Decomposition into special cubes and its application to quasi-subanalytic geometry, Ann. Polon. Math. 96 (1) (2009), 65-74.

[20] - Quantifier elimination, valuation property and preparation theorem in quasianalytic geometry via transformation to normal crossings, Ann. Polon. Math. 96(3) (2009), 247-282.

[21] - Rectilinearization of functions definable by a Weierstrass system and its applications, Ann. Polon. Math. 99 (2) (2010), 129-141.

[22] —, Quasianalytic structures revisited: quantifier elimination, valuation property and rectilinearization of functions, IMUJ Preprint 2012/02, RAAG Preprint 340 (2012).

[23] —, On the singular locus of sets definable in a quasianalytic structure, Adv. Geom. 13 (2013), 619-632.

[24] -, On division of quasianalytic function germs, Int. J. Math. 24 (13) (2013), 1-5.

[25] - , A counterexample concerning quantifier elimination in quasianalytic structures, IMUJ Preprint 2012/04, RAAG Preprint 343 (2012), arXiv:1310.1303 [math.AG] (2013). 
[26] J.-P. Rolin, T. Servi, Quantifier elimination and rectilinearization theorems for generalised quasianalytic algebras, arXiv:1303.3724 [math.AG], (2013).

[27] —, P. Speissegger, A.J. Wilkie, Quasianalytic Denjoy-Carleman classes and ominimality, J. Amer. Math. Soc. 16 (2003), 751-777.

[28] C. Roumieu, Ultradistributions définies sur $\mathbb{R}^{n}$ et sur certaines classes de variétés differentiables, J. Analyse Math. 10 (1962-62), 153-192.

[29] W. Rudin, Real and Complex Analysis, McGraw-Hill, New York, 1987.

[30] V. Thilliez, On quasianalytic local rings, Expo. Math. 26 (1) (2008), 1-23.

[31] —, On the non-extendability of quasianalytic germs, arXiv:1006.4171 [math.CA], v.1 (2010).

[32] H. Whitney, Differentiable even functions, Duke Math. J. 10 (1943), 159-160.

Institute of Mathematics

Faculty of Mathematics and Computer Science

Jagiellonian University

ul. Profesora Łojasiewicza 6

30-348 Kraków, Poland

e-mail: nowak@im.uj.edu.pl 